\title{
Sleep-wake behaviour during the first 12 months of life and associated factors: a systematic review
}

\section{Cláudia Castro Dias \& Bárbara Figueiredo}

To cite this article: Cláudia Castro Dias \& Bárbara Figueiredo (2019): Sleep-wake behaviour during the first 12 months of life and associated factors: a systematic review, Early Child Development and Care

To link to this article: https://doi.org/10.1080/03004430.2019.1582034

曲 Published online: 06 Mar 2019.

Submit your article to this journal 정

View Crossmark data \lceil 


\title{
Sleep-wake behaviour during the first 12 months of life and associated factors: a systematic review
}

\author{
Cláudia Castro Dias (D) and Bárbara Figueiredo
}

School of Psychology, University of Minho, Braga, Portugal

\begin{abstract}
This paper aims to provide a systematic review of the literature on the associated factors with infant sleep-wake behaviour during the first 12 months of life, namely (1) the factors positively and negatively associated with sleep-wake behaviour and (2) the factors positively and negatively affected by sleep-wake behaviour. This systematic review was based on a standard protocol constructed according with the Preferred Reporting Items for Systematic Reviews and Meta-Analysis (PRISMA) statement and included 146 papers. Two typologies of factors were found in the literature: factors positively and negatively associated with infant sleep-wake behaviour and effects of sleep-wake behaviour. Different factors were identified as being positively and negatively associated with the different sleep-wake behaviours and positively and negatively affected by the different sleep-wake behaviours during the first 12 months of life. Some inconsistent results could be explained by methodological differences between the studies.
\end{abstract}

\section{ARTICLE HISTORY}

Received 19 December 2018 Accepted 10 February 2019

\section{KEYWORDS}

Sleep-wake behaviour; infant; positive factors; negative factors

Literature has been shown that infant sleep-wake behaviour is associated with several factors: infant factors, namely temperament (Kaley, Reid, \& Flynn, 2012; Sorondo \& Reeb-Sutherland, 2015) and parental factors, namely parental psychopathology (Sadeh, Tikotzky, \& Scher, 2010). Moreover, a recent review pointed for a bidirectional association, arguing that parental factors influence and are influenced by infant sleep-wake behaviour, within the context of a transactional model (Sadeh et al., 2010). The transactional model argues that the bidirectional associations between parental factors and infant sleep-wake behaviors are complex and dynamic (Sadeh et al., 2010).

Several changes and high individual stability have been observed in infant sleep wake-behaviour during the first months (Figueiredo, Dias, Pinto, \& Field, 2016), being imperative to identify the factors that could be associated with sleep-wake behaviour during the first 12 months of life. The identification of these factors could help to the prevention and early intervention on infant's sleep problems and to guide future research on infant sleep-wake behaviour.

To our knowledge, there is no systematic review in the literature identifying the factors positively and negatively associated with sleep-wake behaviour during the first 12 months of life. This paper aims to provide a systematic review of the literature on the associated factors with infant sleepwake behaviour during the first 12 months of life, namely (1) the factors positively and negatively associated with sleep-wake behaviour and (2) the factors positively and negatively affected by sleep-wake behaviour. 


\section{Materials and methods}

This systematic review was based on a standard protocol constructed according with the Preferred Reporting Items for Systematic Reviews and Meta-Analysis (PRISMA) statement (Moher, Liberati, Tetzlaff, \& Altman, 2009).

\section{Literature search}

The authors performed a literature search to identify manuscripts referring infant sleep-wake behaviour published between June 1996 and June 2016. The search covered three databases: MEDLINE, ISIs Web of Knowledge, and PsycINFO using the keywords: sleep AND infant OR baby. Considering the spoken languages by the two authors, English, Portuguese, Spanish, Italian, and French written articles were included. The first author performed the electronic search and the second author independently replicated it. To select potentially relevant papers, the two authors independently analyzed the titles and the abstracts $(n=8610)$ obtained in the electronic search and excluded the manuscripts that did not study factors associated with infant sleep-wake behaviour $(n=8244)$. The manuscripts whose abstracts assessed factors associated with infant sleep-wake behaviour $(n=367)$ were then independently analyzed for inclusion/exclusion criteria by the two authors, leading to the exclusion of 220 manuscripts. Through all the literature search process, the two authors reached consensus through discussion.

\section{Inclusion and exclusion criteria}

This systematic review included only original empirical articles addressing factors associated with sleep-wake behaviour during the first 12 months of life. The authors used the following inclusion criteria: (a) original studies; (b) assessing factors associated with sleep-wake behaviour; (c) during the first 12 months of life. Studies were excluded based on the following exclusion criteria: (a) non-original research (e.g. literature reviews, systematic reviews, or meta-analysis); (b) studies assessing infants above 12 months or studies that did not indicate infant's age; (c) sleep interventions or clinical trials; (d) case reports or single case studies.

\section{Quality assessment}

All included studies were scored for quality, based on the system of Downs and Black (Downs \& Black, 1998), a Quality Index that evaluates the methodological quality of randomized and non-randomized studies. The Quality Index is comprised of 27 items that are aggregated in 5 sub-scales. Fourteen items, from the total 27 , were selected to score all the studies that were included in this systematic review, with a maximum possible score of 15 . Manuscripts scoring $>10$ in the Quality Index were considered good, those scored between seven and 10 were considered moderate, and those scoring < seven were considered poor.

\section{Data extraction}

One author extracted the data from the studies to 2 data sheets (Tables 1 and 2) and the other author reviewed the data. The data sheets were organized according with (1) factors typology, namely: factors positively and negatively associated with infant sleep-wake behaviour and factors positively and negatively affected by infant sleep-wake behaviour and (2) the publication year.

This systematic review included the different sleep-wake behaviors studied in the literature: sleep duration (number of hours that infants spend sleeping), Wake After Sleep Onset (WASO) (number of hours that infants spend awake during the night), sleep periods (number of sleep episodes) and night wakings (number of wakings during the night), sleep latency (time infants take to fall asleep), longest 


\begin{tabular}{|c|c|c|c|c|c|c|c|}
\hline First author Year of publication & Country & Design & Associated factor & $\begin{array}{c}N \\
\text { Age } \\
\text { (months) }\end{array}$ & Sleep measure & Sleep-wake behaviour & Main result \\
\hline \multicolumn{8}{|c|}{ Factors positively associated with infant sleep-wake behaviour during the first 12 months of life } \\
\hline Blair et al. (2012) & UK & L & Birth order & $\begin{array}{l}11478 \\
6 \text { months }\end{array}$ & Q & SD $(24 \mathrm{~h})$ & SD (24 h) at 6 months not associated with number of siblings \\
\hline Kaley et al. (2012) & UK & CS & Birth order & $\begin{array}{l}77 \\
1-2 \text { months }\end{array}$ & $\mathrm{D}$ & $\begin{array}{l}\text { SD (24 h, day, night), } \\
\text { NW }\end{array}$ & $\begin{array}{l}\uparrow \text { SD ( } 24 \mathrm{~h} \text {, day) at } 1-2 \text { months in non-first-time infants } \\
\text { NW not associated with birth order }\end{array}$ \\
\hline $\begin{array}{l}\text { Kozyrskyj, Kendall, Zubrick, } \\
\text { Newnham, and Sly (2009) }\end{array}$ & Australia & $\mathrm{L}$ & Birth order & $\begin{array}{l}2398 \\
12 \text { months }\end{array}$ & Q & NW & $\uparrow \mathrm{NW}$ at 12 months associated with $\uparrow$ siblings \\
\hline $\begin{array}{l}\text { Santos, Mota, and Matijasevich } \\
\text { (2008) }\end{array}$ & Brazil & $\mathrm{L}$ & Birth order & $\begin{array}{l}3907 \\
0 \text { and } 12 \text { months }\end{array}$ & I & NW & $\uparrow N W$ at 12 months in non-first-born infants \\
\hline $\begin{array}{l}\text { So, Michael Adamson, and Horne } \\
\text { (2007) }\end{array}$ & Australia & $\mathrm{L}$ & Birth order & $\begin{array}{l}20 \\
1,2,3,4,5,6,7 \\
8,9,10,11 \text {, and } \\
12 \text { months }\end{array}$ & $\begin{array}{l}\text { Combined } \\
\text { measures: A and } \\
\text { D }\end{array}$ & $\begin{array}{l}\text { SD (24 h, day, night), } \\
\text { NW }\end{array}$ & $\begin{array}{l}\text { SD ( } 24 \text { h, day, night) and NW at } 1,2,3,4,5,6,8,9,10,11 \text {, and } \\
12 \text { months not associated with birth order } \\
\downarrow \text { SD (night) at } 7 \text { months (measured by D) in non-first-born } \\
\text { infants }\end{array}$ \\
\hline Quillin (1997) & USA & CS & Birth order & $\begin{array}{l}44 \\
1 \text { month }\end{array}$ & $\mathrm{D}$ & $\begin{array}{l}\text { SD ( } 24 \mathrm{~h}, \text { night }), N W, \\
\text { SP ( } 24 \mathrm{~h})\end{array}$ & $\begin{array}{l}\text { SD ( } 24 \mathrm{~h} \text {, night), NW, and SP }(24 \mathrm{~h}) \text { at } 1 \text { months not } \\
\text { associated with birth order }\end{array}$ \\
\hline Blair et al. (2012) & UK & $\mathrm{L}$ & Ethnicity & $\begin{array}{l}11478 \\
6 \text { months }\end{array}$ & Q & SD $(24 \mathrm{~h})$ & $\downarrow \mathrm{SD}(24 \mathrm{~h})$ at 6 months in non-white infants \\
\hline $\begin{array}{l}\text { Santos, Matijasevich, and } \\
\text { Domingues (2012) }\end{array}$ & Brazil & CS & Ethnicity & $\begin{array}{l}885 \\
3 \text { months }\end{array}$ & I & NW & $\uparrow N W$ at 3 months in non-white infants \\
\hline $\begin{array}{l}\text { Nevarez, Rifas-Shiman, Kleinman, } \\
\text { Gillman, and Taveras (2010) }\end{array}$ & USA & $\mathrm{L}$ & Ethnicity & $\begin{array}{l}1676 \\
6 \text {, and } 12 \text { months }\end{array}$ & Q & SD (24 h) & $\downarrow \mathrm{SD}(24 \mathrm{~h})$ at 6 and 12 months in non-white infants \\
\hline Bathory et al. (2016) & USA & CS & Parental education & $\begin{array}{l}557 \\
9 \text { months }\end{array}$ & Q & SD (day, night) & $\begin{array}{l}\downarrow \text { SD (night) associated with } \downarrow \text { parental health literacy } \\
\text { SD (day) not associated with parental health literacy }\end{array}$ \\
\hline Blair et al. (2012) & UK & $\mathrm{L}$ & Parental education & $\begin{array}{l}11478 \\
6 \text { months }\end{array}$ & Q & SD (24 h) & $\begin{array}{l}\text { SD }(24 \mathrm{~h}) \text { at } 6 \text { months not associated with maternal } \\
\text { education and paternal social class }\end{array}$ \\
\hline Kozyrskyj et al. (2009) & Australia & $\mathrm{L}$ & Parental education & $\begin{array}{l}2398 \\
12 \text { months }\end{array}$ & Q & NW & NW at 12 months not associated with maternal education \\
\hline Nevarez et al. (2010) & USA & $\mathrm{L}$ & Parental education & $\begin{array}{l}1676 \\
6 \text {, and } 12 \text { months }\end{array}$ & Q & SD (24 h) & $\begin{array}{l}\downarrow \mathrm{SD}(24 \mathrm{~h}) \text { at } 6 \text { and } 12 \text { months associated with } \downarrow \text { maternal } \\
\text { education }\end{array}$ \\
\hline $\begin{array}{l}\text { Palmstierna, Sepa, and Ludvigsson } \\
\text { (2008) }\end{array}$ & Sweden & $\mathrm{L}$ & Parental education & $\begin{array}{l}16467 \\
12 \text { months }\end{array}$ & Q & NW & $\begin{array}{l}\uparrow \mathrm{NW} \text { at } 12 \text { months associated with } \uparrow \text { parental theoretical } \\
\text { education }\end{array}$ \\
\hline Orsi et al. (2015) & Brazil & $\mathrm{L}$ & NICU characteristic & $\begin{array}{l}13 \\
0 \text { months }\end{array}$ & $P$ & $\begin{array}{l}\text { SD ( } 24 \mathrm{~h} \text {, day, and } \\
\text { night) }\end{array}$ & $\begin{array}{l}\uparrow \mathrm{SD} \text { ( } 24 \mathrm{~h} \text { and day) associated with reduced sensory and } \\
\text { environmental stimuli } \\
\text { SD (night) not associated with sensory and environmental } \\
\text { stimuli }\end{array}$ \\
\hline Touchette et al. (2013) & Canada & $\mathrm{L}$ & $\begin{array}{l}\text { Genetic/ } \\
\text { environment } \\
\text { (twins) }\end{array}$ & $\begin{array}{l}995 \\
6 \text { months }\end{array}$ & Q & SD (day, night) & $\begin{array}{l}\uparrow S D \text { (night) at } 6 \text { months associated with genetic influences } \\
\text { SD (day) at } 6 \text { months associated with environmental } \\
\text { influences }\end{array}$ \\
\hline $\begin{array}{l}\text { Tourula, Isola, Hassi, Bloigu, and } \\
\text { Rintamäki (2010) }\end{array}$ & Finland & CS & Environment & $\begin{array}{l}34 \\
3 \text { months }\end{array}$ & 0 & SD (day) & $\uparrow S D$ (day) at 3 months in outdoor environment \\
\hline
\end{tabular}




\begin{tabular}{|c|c|c|c|c|c|c|c|}
\hline First author Year of publication & Country & Design & Associated factor & $\begin{array}{c}N \\
\text { Age } \\
\text { (months) }\end{array}$ & Sleep measure & Sleep-wake behaviour & Main result \\
\hline Bhat et al. (2006) & UK & CS & Sleep position & $\begin{array}{l}24 \\
0 \text { months }\end{array}$ & $P$ & SD (day), SP (day), SE & $\begin{array}{l}\uparrow \mathrm{SD} \text { (day), } \downarrow \text { SP (day), and } \uparrow \mathrm{SE} \text { at } 0 \text { months associated with } \\
\text { prone position }\end{array}$ \\
\hline $\begin{array}{l}\text { Utkun, Öndoğan, Yalaz, and } \\
\text { Yıldırım Sözmen (2015) }\end{array}$ & Turkey & CS & Clothes' fabrics & $\begin{array}{l}8 \\
6-12 \text { months }\end{array}$ & NS & SD (night) & $\begin{array}{l}\uparrow \text { SD (night) at 6-12 months associated with wearing Ö4 } \\
\text { fabric }\end{array}$ \\
\hline $\begin{array}{l}\text { Brand, Furlano, Sidler, Schulz, and } \\
\text { Holsboer-Trachsler (2011) }\end{array}$ & Switzerland & L & Cortisol & $\begin{array}{l}16 \\
2 \text { and } 3 \text { months }\end{array}$ & A & $\begin{array}{l}\text { SD (day, night), A } \\
\text { (day, night) }\end{array}$ & $\begin{array}{l}\uparrow \mathrm{SD} \text { (night) and } \uparrow \mathrm{NW} \text { at } 3 \text { months associated with } \uparrow \text { cortisol } \\
\text { secretion in infants with colic }\end{array}$ \\
\hline Lucas-Thompson et al. (2009) & USA & L & Cortisol & $\begin{array}{l}92 \\
6 \text { and } 12 \text { months }\end{array}$ & Q & NW & $\begin{array}{l}\uparrow N W \text { at } 6 \text { and } 12 \text { months associated with } \uparrow \text { cortisol response } \\
\text { to stress at } 6 \text { and } 12 \text { months }\end{array}$ \\
\hline $\begin{array}{l}\text { Larson, White, Cochran, Donzella, } \\
\text { and Gunnar (1998) }\end{array}$ & USA & CS & Cortisol & $\begin{array}{l}78 \\
2-4 \text { months }\end{array}$ & D & SD (night) & $\begin{array}{l}\uparrow S D \text { (night) at } 2-4 \text { months associated with early morning } \\
\text { peak in cortisol levels }\end{array}$ \\
\hline Shinohara and Kodama (2011) & Japan & CS & Melatonin & $\begin{array}{l}67 \\
3-5 \text { months }\end{array}$ & $\mathrm{D}$ & $\begin{array}{l}\text { SD (night), NW, SP } \\
\text { (day), LSP (24 h) }\end{array}$ & $\begin{array}{l}\uparrow \text { SD (night) at 3-5 months associated with } \downarrow \text { morning } \\
\text { melatonin levels at 3-5 months }\end{array}$ \\
\hline Pennestri et al. (2015) & Canada & $\mathrm{L}$ & Attachment & $\begin{array}{l}138 \\
6,12 \text { months }\end{array}$ & Q & $\begin{array}{l}\text { SD (night), NW, SL } \\
\text { (night), LSP (night), } \\
\text { BT }\end{array}$ & $\begin{array}{l}\downarrow \text { SD (night), } \uparrow \text { NW and } \uparrow \text { BT at } 6 \text { and } 12 \text { months in } \\
\text { disorganized children than in secure and ambivalent } \\
\text { children } \\
\downarrow \text { LSP (night) at } 12 \text { months in disorganized children than in } \\
\text { secure and ambivalent children } \\
\text { SL (night) not associated with attachment }\end{array}$ \\
\hline $\begin{array}{l}\text { Zentall, Braungart-Rieker, Ekas, } \\
\text { and Lickenbrock (2012) }\end{array}$ & USA & L & Attachment & $\begin{array}{l}46 \\
7,12 \text { months }\end{array}$ & Q & NW & $\begin{array}{l}\downarrow N W \text { from } 7 \text { to } 12 \text { and } 14 \text { months in infants with secure } \\
\text { attachment } \\
\text { NW at } 7 \text { months not associated with attachment }\end{array}$ \\
\hline Scher (2001a) & Israel & CS & Attachment & $\begin{array}{l}94 \\
12 \text { months }\end{array}$ & A & $\begin{array}{r}\text { SD (night), NW, LSP } \\
\text { (night), SL (night) }\end{array}$ & $\begin{array}{l}\text { SD (night), NW, LSP (night), and SL (night) at } 12 \text { months not } \\
\text { associated with attachment style }\end{array}$ \\
\hline $\begin{array}{l}\text { de Graag, Cox, Hasselman, Jansen, } \\
\text { and de Weerth (2012) }\end{array}$ & The Netherlands & $\mathrm{L}$ & $\begin{array}{l}\text { Mother-infant } \\
\text { relationship }\end{array}$ & $\begin{array}{l}84 \\
1 \text { and } 5 \text { months }\end{array}$ & D & LSP $(24 \mathrm{~h})$ & $\begin{array}{l}\uparrow \text { LSP }(24 \mathrm{~h}) \text { from } 1 \text { to } 5 \text { months associated with } \uparrow \text { flexible } \\
\text { mother-infant interactions at } 5 \text { months }\end{array}$ \\
\hline $\begin{array}{l}\text { Schwichtenberg, Anders, } \\
\text { Vollbrecht, and Poehlmann } \\
\text { (2011) }\end{array}$ & USA & $\mathrm{L}$ & $\begin{array}{l}\text { Mother-infant } \\
\text { relationship }\end{array}$ & $\begin{array}{l}134 \\
4 \text { and } 9 \text { months }\end{array}$ & $\mathrm{D}$ & SD (day), SP (day) & $\begin{array}{l}\uparrow \mathrm{SD} \text { (day) at } 9 \text { months associated with } \uparrow \text { maternal negative } \\
\text { affect and behaviour at } 4 \text { and } 9 \text { months } \\
\uparrow \mathrm{SP} \text { (day) at } 4 \text { and } 9 \text { months associated with } \uparrow \text { optimal } \\
\text { maternal interactive behaviors at } 24 \text { months }\end{array}$ \\
\hline Scher (2001b) & Israel & CS & $\begin{array}{l}\text { Mother-infant } \\
\text { relationship }\end{array}$ & $\begin{array}{l}37 \\
12 \text { months }\end{array}$ & A & SD (night), NW, SE, BT & $\begin{array}{l}\uparrow \mathrm{NW} \text { at } 12 \text { months associated with } \uparrow \text { mother-infant } \\
\text { relationship } \\
\uparrow \mathrm{SE} \text { at } 12 \text { months associated with } \uparrow \text { infant responsiveness, } \\
\text { but not with other mother-infant interaction dimensions } \\
\mathrm{SD} \text { (night) and BT at } 12 \text { months not associated with mother- } \\
\text { infant interaction }\end{array}$ \\
\hline \multicolumn{8}{|c|}{ Factors negatively associated with infant sleep-wake behaviour during the first 12 months of life } \\
\hline $\begin{array}{l}\text { Saenz, Yaugher, and Alexander } \\
\text { (2015) }\end{array}$ & USA & $\mathrm{L}$ & Gender & $\begin{array}{l}47 \\
3-4 \text { months }\end{array}$ & A & SD (night), SE & SD (night) and SE at 3-4 months not associated with gender \\
\hline Blair et al. (2012) & UK & $\mathrm{L}$ & Gender & $\begin{array}{l}11478 \\
6 \text { months }\end{array}$ & Q & SD (24 h) & $\downarrow S D(24 \mathrm{~h})$ at 6 months in boys \\
\hline Kaley et al. (2012) & UK & CS & Gender & $\begin{array}{l}77 \\
1-2 \text { months }\end{array}$ & $\mathrm{D}$ & $\begin{array}{l}\text { SD (24 h, day, night), } \\
\text { NW }\end{array}$ & $\begin{array}{l}\uparrow N W \text { at } 1-2 \text { months in boys } \\
\text { SD ( } 24 \text { h, day, night) not associated with gender }\end{array}$ \\
\hline
\end{tabular}




\begin{tabular}{|c|c|c|c|c|c|c|c|}
\hline Santos et al. (2012) & Brazil & CS & Gender & $\begin{array}{l}885 \\
3 \text { months }\end{array}$ & I & NW & $\uparrow N W$ at 3 months in boys \\
\hline Lampl and Johnson (2011) & USA & L & Gender & $\begin{array}{l}23 \\
0-12 \text { months }\end{array}$ & $\mathrm{D}$ & $\begin{array}{l}\text { SD }(24 \mathrm{~h}), \text { SP }(24 \mathrm{~h}) \\
\quad \operatorname{LSP}(24 \mathrm{~h})\end{array}$ & $\begin{array}{l}\uparrow S P(24 h) \text { and } \downarrow \text { LSP }(24 h) \text { at } 0-12 \text { months in boys } \\
\text { SD }(24 h) \text { not associated with gender }\end{array}$ \\
\hline Kozyrskyj et al. (2009) & Australia & $\mathrm{L}$ & Gender & $\begin{array}{l}2398 \\
12 \text { months }\end{array}$ & Q & NW & NW at 12 months not associated with gender \\
\hline Santos et al. (2008) & Brazil & L & Gender & $\begin{array}{l}3907 \\
0 \text { and } 12 \text { months }\end{array}$ & I & NW & $\uparrow \mathrm{NW}$ at 12 months more in boys \\
\hline $\begin{array}{l}\text { Anuntaseree, Mo-Suwan, } \\
\text { Vasiknanonte, Kuasirikul, and } \\
\text { Choprapawan (2008) }\end{array}$ & Thailand & CS & Gender & $\begin{array}{l}3172 \\
3 \text { months }\end{array}$ & I & NW & $\uparrow N W$ at 3 months in boys \\
\hline So et al. (2007) & Australia & $\mathrm{L}$ & Gender & $\begin{array}{l}20 \\
1,2,3,4,5,6,7 \\
8,9,10,11 \text {, and } \\
12 \text { months }\end{array}$ & $\begin{array}{l}\text { Combined } \\
\text { measures: A and } \\
\text { D }\end{array}$ & $\begin{array}{l}\text { SD ( } 24 \text { h, day, night), } \\
\text { WASO, SP (day), LSP } \\
\text { (night) }\end{array}$ & $\begin{array}{l}\downarrow \text { SD (day) at } 1 \text { (measured by } A \text { and D) and } 2 \text { (measured by A) } \\
\text { months in boys } \\
\text { SD (day) at } 3,4,5,6,7,8,9,10,11 \text {, and } 12 \text { months not } \\
\text { associated with gender } \\
\text { SD ( } 24 \text { h, night), WASO, and LSP (night) at } 1,2,3,4,5,6,7,8 \text {, } \\
9,10,11 \text {, and } 12 \text { months not associated with gender }\end{array}$ \\
\hline Thomas and Foreman (2005) & USA & CS & Gender & $\begin{array}{l}37 \\
1-2 \text { months }\end{array}$ & $\mathrm{D}$ & $\begin{array}{l}\text { SD }(24 \mathrm{~h}), \text { SP }(24 \mathrm{~h}) \\
\quad \text { LSP }(24 \mathrm{~h})\end{array}$ & $\begin{array}{l}\downarrow \mathrm{SD}(24 \mathrm{~h}) \text { at } 1-2 \text { months in boys } \\
\text { SP }(24 \mathrm{~h}), \mathrm{LSP}(24 \mathrm{~h}) \text { at } 1-2 \text { months not associated with } \\
\text { gender }\end{array}$ \\
\hline $\begin{array}{l}\text { Goodlin-Jones, Burnham, Gaylor, } \\
\text { and Anders (2001) }\end{array}$ & USA & CS & Gender & $\begin{array}{l}80 \\
3,6,9 \text {, or } 12 \\
\text { months }\end{array}$ & V & NW, LSP (night) & $\begin{array}{l}\uparrow \text { LSP (night) in girls } \\
\text { NW not associated with gender }\end{array}$ \\
\hline $\begin{array}{l}\text { Bach, Telliez, Leke, and Libert } \\
\text { (2000) }\end{array}$ & France & CS & Gender & $\begin{array}{l}38 \\
1 \text { month }\end{array}$ & EEG & SD (24 h), LSP (24 h) & $\downarrow S D(24 \mathrm{~h})$, and $\downarrow$ LSP $(24 \mathrm{~h})$ at 1 month in boys \\
\hline Blair et al. (2012) & UK & $\mathrm{L}$ & $\begin{array}{l}\text { Parents' socio- } \\
\text { demographic } \\
\text { factors }\end{array}$ & $\begin{array}{l}11478 \\
6 \text { months }\end{array}$ & Q & SD $(24 \mathrm{~h})$ & $\downarrow \mathrm{SD}(24 \mathrm{~h})$ at 6 months in infants of older mothers \\
\hline Kozyrskyj et al. (2009) & Australia & $\mathrm{L}$ & $\begin{array}{l}\text { Parents' socio- } \\
\text { demographic } \\
\text { factors }\end{array}$ & $\begin{array}{l}2398 \\
12 \text { months }\end{array}$ & Q & NW & $\begin{array}{l}\uparrow N W \text { at } 12 \text { months in infants of mothers older than } 20 \text { years } \\
N W \text { at } 12 \text { months not associated with maternal marital status }\end{array}$ \\
\hline Nevarez et al. (2010) & USA & $\mathrm{L}$ & $\begin{array}{l}\text { Parents' socio- } \\
\text { demographic } \\
\text { factors }\end{array}$ & $\begin{array}{l}1676 \\
6 \text {, and } 12 \text { months }\end{array}$ & Q & SD $(24 \mathrm{~h})$ & $\begin{array}{l}\downarrow \mathrm{SD}(24 \mathrm{~h}) \text { at } 6 \text { and } 12 \text { months associated with infants of } \\
\text { single/divorced parents }\end{array}$ \\
\hline Palmstierna et al. (2008) & Sweden & $\mathrm{L}$ & $\begin{array}{l}\text { Parents' socio- } \\
\text { demographic } \\
\text { factors }\end{array}$ & $\begin{array}{l}16467 \\
12 \text { months }\end{array}$ & Q & NW & $\begin{array}{l}\uparrow \mathrm{NW} \text { at } 12 \text { months associated with non-Swedish parents and } \\
\text { marginally associated with single parent at birth }\end{array}$ \\
\hline Tomalski et al. (2013) & UK & CS & $\begin{array}{l}\text { Socio-economic } \\
\text { factors }\end{array}$ & $\begin{array}{l}45 \\
6-9 \text { months }\end{array}$ & Q & $\begin{array}{l}\text { SD ( } 24 \text { h, day, night), } \\
\text { WASO, NW }\end{array}$ & $\begin{array}{l}\text { SD ( } 24 \mathrm{~h} \text {, day, night), WASO, and NW not associated with } \\
\text { income }\end{array}$ \\
\hline Nevarez et al. (2010) & USA & L & $\begin{array}{l}\text { Socio-economic } \\
\text { factors }\end{array}$ & $\begin{array}{l}1676 \\
6 \text { and } 12 \text { months }\end{array}$ & Q & SD $(24 \mathrm{~h})$ & $\begin{array}{l}\downarrow \text { SD }(24 \mathrm{~h}) \text { at } 6 \text { and } 12 \text { months associated with } \downarrow \text { household } \\
\text { incomes, and } \uparrow \text { household members }\end{array}$ \\
\hline Kozyrskyj et al. (2009) & Australia & $\mathrm{L}$ & $\begin{array}{l}\text { Socio-economic } \\
\text { factors }\end{array}$ & $\begin{array}{l}2398 \\
12 \text { months }\end{array}$ & Q & NW & $\begin{array}{l}\text { NW at } 12 \text { months not associated with financial problems and } \\
\text { income }\end{array}$ \\
\hline Santos et al. (2008) & Brazil & $\mathrm{L}$ & $\begin{array}{l}\text { Socio-economic } \\
\text { factors }\end{array}$ & $\begin{array}{l}3907 \\
0 \text { and } 12 \text { months }\end{array}$ & I & NW & $\begin{array}{l}\uparrow \mathrm{NW} \text { at } 12 \text { months in infants whose mothers were employed } \\
\text { during pregnancy }\end{array}$ \\
\hline
\end{tabular}




\begin{tabular}{|c|c|c|c|c|c|c|c|}
\hline First author Year of publication & Country & Design & Associated factor & $\begin{array}{c}N \\
\text { Age } \\
\text { (months) }\end{array}$ & Sleep measure & Sleep-wake behaviour & Main result \\
\hline $\begin{array}{l}\text { Fouts, Roopnarine, and Lamb } \\
\text { (2007) }\end{array}$ & USA & CS & $\begin{array}{l}\text { Socio-economic } \\
\text { factors }\end{array}$ & $\begin{array}{l}62 \\
3-4 \text { months }\end{array}$ & 0 & $\begin{array}{l}\text { SD (day), SP (day), LSP } \\
\text { (day) }\end{array}$ & $\begin{array}{l}\uparrow \text { SP (day) and } \downarrow \text { LSP (day) at 3-4 months in infants from } \downarrow \\
\text { socioeconomic status } \\
\text { SD (day) at 3-4 months not associated with socioeconomic } \\
\text { status }\end{array}$ \\
\hline $\begin{array}{l}\text { Cohen, Atun-Einy, and Scher } \\
\text { (2012) }\end{array}$ & Israel & CS & Environment & $\begin{array}{l}34 \\
7 \text { months }\end{array}$ & A & $\begin{array}{l}\text { SD (night), WASO, } \\
\text { NW, BT, WT }\end{array}$ & $\begin{array}{l}\uparrow \text { BT at } 7 \text { months in winter months } \\
\text { SD (night), WASO, and NW and WT at } 7 \text { months not } \\
\text { associated with seasonal effects }\end{array}$ \\
\hline Bottino et al. (2012) & USA & CS & Environment & $\begin{array}{l}1226 \\
12 \text { months }\end{array}$ & Q & SD (24 h) & $\begin{array}{l}\downarrow \text { SD (24-hour) at } 12 \text { months associated with } \uparrow \text { urban } \\
\text { environments }\end{array}$ \\
\hline Harrison (2004) & UK & $\mathrm{L}$ & Environment & $\begin{array}{l}66 \\
1,2,3 \text { months }\end{array}$ & $\begin{array}{l}\text { Combined } \\
\text { measures: D and } \\
\text { A }\end{array}$ & SD (night) & $\begin{array}{l}\downarrow S D \text { (night) at } 1 \text { month associated with } \downarrow \text { exposure to light } \\
\text { during the afternoon }\end{array}$ \\
\hline Kozyrskyj et al. (2009) & Australia & $\mathrm{L}$ & Environment & $\begin{array}{l}2398 \\
12 \text { months }\end{array}$ & Q & NW & $\begin{array}{l}\uparrow \mathrm{NW} \text { at } 12 \text { months associated with mothers not smoking at } \\
12 \text { months } \\
\text { NW at } 12 \text { months not associated with exposure to stressful } \\
\text { events }\end{array}$ \\
\hline Santos et al. (2012) & Brazil & CS & $\begin{array}{r}\text { Gestational } \\
\text { exposure }\end{array}$ & $\begin{array}{l}885 \\
3 \text { months }\end{array}$ & 1 & NW & $\begin{array}{l}\text { NW at } 3 \text { months not associated with gestational exposure to } \\
\text { caffeine } \\
\uparrow N W \text { at } 3 \text { months in infants exposed to smoke and alcohol } \\
\text { during pregnancy }\end{array}$ \\
\hline Kozyrskyj et al. (2009) & Australia & $\mathrm{L}$ & $\begin{array}{r}\text { Gestational } \\
\text { exposure }\end{array}$ & $\begin{array}{l}2398 \\
12 \text { months }\end{array}$ & Q & NW & $\begin{array}{l}\uparrow \mathrm{NW} \text { at } 12 \text { months associated with } \uparrow \text { stressful events during } \\
\text { early pregnancy and no exposure to smoke during } \\
\text { pregnancy }\end{array}$ \\
\hline $\begin{array}{l}\text { Lehnkering, Korte, and Siegmund } \\
\text { (2009) }\end{array}$ & Germany & CS & $\begin{array}{r}\text { Gestational } \\
\text { exposure }\end{array}$ & $\begin{array}{l}22 \\
0 \text { months }\end{array}$ & $\begin{array}{l}\text { Combined } \\
\text { measures: } A \text { and } \\
\text { D }\end{array}$ & SD (24 h) & $\begin{array}{l}\text { SD }(24 \mathrm{~h}) \text { at } 0 \text { months not associated with gestational } \\
\text { exposure to smoke }\end{array}$ \\
\hline $\begin{array}{l}\text { Hanft, Burnham, Goodlin-Jones, } \\
\text { and Anders (2006) }\end{array}$ & USA & L & $\begin{array}{r}\text { Gestational } \\
\text { exposure }\end{array}$ & $\begin{array}{l}34 \\
1,3,6,9 \text {, and } 12 \\
\text { months }\end{array}$ & V & $\begin{array}{l}\text { SD (night), WASO, } \\
\text { NW, LSP (night) }\end{array}$ & $\begin{array}{l}\downarrow S D \text { (night) at } 3,6 \text {, and } 12 \text { months and } \downarrow \text { LSP (night) at } 6 \\
\text { months in infants exposed to substances during pregnancy } \\
\text { WASO and NW at } 1,3,6,9 \text {, and } 12 \text { months, SD (night) at } 1 \\
\text { and } 9 \text { months and LSP (night) at } 1,3,9 \text {, and } 12 \text { months not } \\
\text { associated with gestational exposure to substances }\end{array}$ \\
\hline $\begin{array}{l}\text { Korte, Hoehn, and Siegmund } \\
\text { (2004) }\end{array}$ & Germany & L & Delivery mode & $\begin{array}{l}57 \\
0 \text { months }\end{array}$ & A & $\begin{array}{l}\text { SD ( } 24 \text { h, day, night), } \\
\text { SP (day) }\end{array}$ & $\begin{array}{l}\text { SD ( } 24 \mathrm{~h} \text {, day, night) at } 0 \text { months not associated with delivery } \\
\text { mode } \\
\uparrow \mathrm{SP} \text { (day) at } 0 \text { months in infants born by planed C-section }\end{array}$ \\
\hline $\begin{array}{l}\text { Huang, Paiva, Hsu, Kuo, and } \\
\text { Guilleminault (2014) }\end{array}$ & China & CS & Gestational age & $\begin{array}{l}191 \\
6 \text { months }\end{array}$ & Q & $\begin{array}{l}\text { SD (day, night) } \\
\text { NW }\end{array}$ & $\uparrow \mathrm{SD}$ (day, night) and $\uparrow \mathrm{NW}$ at 6 months in preterm infants \\
\hline Guyer et al. (2015) & Switzerland & L & Gestational age & $\begin{array}{l}48 \\
1,3 \text {, and } 6 \\
\text { months }\end{array}$ & D & $\begin{array}{l}\text { SD ( } 24 \text { h, day, night), } \\
\text { LSP (night) }\end{array}$ & $\begin{array}{l}\uparrow \mathrm{SD} \text { (night) and } \uparrow \text { LSP (night) at } 1,3 \text {, and } 6 \text { months in } \\
\text { premature infants } \\
\text { SD ( } 24 \mathrm{~h} \text {, day) at } 1,3 \text {, and } 6 \text { months not associated with } \\
\text { gestational age }\end{array}$ \\
\hline Asaka and Takada (2010) & Japan & CS & Gestational age & $\begin{array}{l}28 \\
12 \text { months }\end{array}$ & A & $\begin{array}{l}\text { SD (24 h, day, night), } \\
\text { WASO, NW }\end{array}$ & $\begin{array}{l}\downarrow S D \text { (night) at } 12 \text { months in preterm infants } \\
\text { SD ( } 24 \mathrm{~h} \text {, day), WASO, and NW at } 12 \text { months not associated } \\
\text { with gestational age }\end{array}$ \\
\hline
\end{tabular}


L Gestational age 2398

Volkovich, Ben-Zion, Karny, Meiri, Israel and Tikotzky (2015)

Brown and Harries (2015)

UK

Huang et al. (2016)

China

CS Feeding method

L $\quad$ Feeding method

(398

153

3 and 6 month

715

6-12 months

524

$1,3,5$, and 8 months

Tikotzky et al. (2015)

Israel

Hughes, Gallagher, and Hannigan Ireland (2015)

Hysing et at (2014)

Norway

Galbally, Lewis, McEgan, Scalzo, Australia and Islam (2013)

Mindell, Du Mond, Tanenbaum, USA

and Gunn (2012)

Ramamurthy et al. (2012) Asia Pacific

Region

L $\quad$ Feeding method

57

3 and 6 months

CS Feeding method

11134

9 months

L $\quad$ Feeding method

55831

6 months

CS Feeding method

3-19 months

L $\quad$ Feeding method

$3,6,12$ months

CS Feeding method 4602

0-12 months

CS Feeding method 77

6-11 months

77

1-2 months

Kaley et al. (2012)

UK

Engler, Hadash, Shehadeh, and Israel Pillar (2012)

Lampl and Johnson (2011)

Nevarez et al. (2010)

Kozyrskyj et al. (2009)

$\begin{array}{ll}\text { CS Feeding method } & 94 \\ & 2-4 \text { months }\end{array}$

23 12 months

L Feeding method 1676

L Feeding method 2398
Combined

NW

measures: $A$ and

D

NW

$\downarrow N W$ at 12 months in preterm infants

$\uparrow N W$ at 3 and 6 months associated with breastfeeding at 3 months

NW at 6-12 months not associated with feeding method

D

SD (24 h, day, night), NW, SP (day), LSP (night)

$\downarrow$ SD (24 h, day) in the first 8 months in exclusively and partially breastfed infants

$\uparrow$ NW in the first 8 months in exclusively and partially breastfed infants than in exclusively formula fed

SD (night), SP (day) and LSP (night) not associated with feeding method

Combined NW measures: $A, D$ and $Q$

0

SD (night), NW

$\uparrow \mathrm{NW}$ at 6 months associated with breastfeeding at 3 and 6 months

$\downarrow$ SD (night) and $\uparrow$ NW at 9 months associated with $\uparrow$ breastfeeding rates

Q NW

Q NW

$\uparrow N W$ at 6 months associated with breastfeeding

NW

$\uparrow N W$ at 3-19 months associated with breastfeeding at 6 months

$0 \quad \mathrm{SD}$ (night), NW, SP $\uparrow \mathrm{NW}$ and $\uparrow \mathrm{SP}$ (day) at 3-12 months associated with (day), LSP (24 h), SL breastfeeding (night)

SD (night), LSP (24 h), and SL (night) at 3-12 months not associated with feeding method

Q SD (24 h, day, night), $\uparrow \mathrm{SD}$ (24-hour, day), $\uparrow \mathrm{NW}, \downarrow \mathrm{LSP}$ (night), $\uparrow \mathrm{SP}$ (day), and $\uparrow \mathrm{BT}$ NW, SP (day), SL from 0 to 6 months in breastfed infants

(night), LSP (night), $\uparrow$ NW, and $\downarrow$ LSP (night) from 6 to 12 months in breastfed BT, WT infants

SD (night) and WT not associated with breastfeeding

Q SD (24 h, day, night), SD (24 h, day, night) and SL (night) at 6-11 months not SL (night) associated with feeding method

D SD (24 h, day, night), $\uparrow N W$ at $1-2$ months in breastfed infants

NW $\quad$ SD ( $24 \mathrm{~h}$, day, night) at $1-2$ months not associated with feeding method

Q SD (night), NW, SL (night)

$\uparrow N W$ and marginally $\uparrow$ SD (night) at 2-4 months associated with breastfeeding

D $\quad$ SD $(24 h), S P(24 h)$

Giated with feeding method

2398 LSP $(24 \mathrm{~h})$

SD $(24 \mathrm{~h})$

$\uparrow$ SP $(24 \mathrm{~h})$ and L ISP (24h) at 0-12 months associated with breastfeeding method at 6 months

NW
SD $(24 \mathrm{~h})$ not associated with feeding method

SD (24 h) at 6 and 12 months not associated with feeding 


\begin{tabular}{|c|c|c|c|c|c|c|c|}
\hline First author Year of publication & Country & Design & Associated factor & $\begin{array}{c}N \\
\text { Age } \\
\text { (months) }\end{array}$ & Sleep measure & Sleep-wake behaviour & Main result \\
\hline Anuntaseree et al. (2008) & Thailand & CS & Feeding method & $\begin{array}{l}3172 \\
3 \text { months }\end{array}$ & 1 & NW & $\uparrow \mathrm{NW}$ at 3 months associated with exclusive breastfeeding \\
\hline $\begin{array}{l}\text { Mindell, Meltzer, Carskadon, and } \\
\text { Chervin (2009) }\end{array}$ & USA & CS & Feeding method & $\begin{array}{l}1473 \\
0-11 \text { months }\end{array}$ & I & $\begin{array}{l}\text { SD (night), NW, SL } \\
\text { (night) }\end{array}$ & $\begin{array}{l}\uparrow N W \text { at } 0-11 \text { months associated with breastfeeding } \\
\text { SD (night) and SL (night) not associated with feeding method }\end{array}$ \\
\hline $\begin{array}{l}\text { Schwichtenberg and Poehlmann } \\
\text { (2009) }\end{array}$ & USA & CS & Feeding method & $\begin{array}{l}128 \\
4 \text { months }\end{array}$ & $\mathrm{D}$ & SD (night), NW & $\begin{array}{l}\downarrow \text { SD (night) and } \uparrow \text { NW at } 4 \text { months in breastfed preterm } \\
\text { infants }\end{array}$ \\
\hline DeLeon and Karraker (2007) & USA & CS & Feeding method & $\begin{array}{l}41 \\
9 \text { months }\end{array}$ & $\mathrm{D}$ & $\begin{array}{l}\text { SD ( } 24 \text { h, day, night), } \\
\text { WASO, NW, SP (day) }\end{array}$ & $\begin{array}{l}\downarrow \text { SD (night), } \uparrow \text { WASO and } \uparrow \text { NW at } 9 \text { months associated with } \\
\text { breastfeeding } \\
\text { SD }(24 \mathrm{~h} \text {, day), and SP (day) not associated with feeding } \\
\text { method }\end{array}$ \\
\hline Cubero et al. (2005) & Spain & CS & Feeding method & $\begin{array}{l}16 \\
3 \text { months }\end{array}$ & A & $\begin{array}{l}\text { SD (night), SL (night), } \\
\text { SE }\end{array}$ & $\begin{array}{l}\uparrow S D \text { (night) and } \uparrow \text { SE at } 3 \text { months associated with } \\
\text { breastfeeding } \\
\text { SL (night) not associated with feeding method }\end{array}$ \\
\hline $\begin{array}{l}\text { Morgan, Lucas, and Fewtrell } \\
\text { (2004) }\end{array}$ & UK & $\mathrm{L}$ & Feeding method & $\begin{array}{l}1694 \\
6,9 \text { months }\end{array}$ & Q & SD (night) & $\begin{array}{l}\downarrow S D \text { (night) at } 9 \text { months associated with } \uparrow \text { breastfed duration } \\
\text { in term infants } \\
\text { SD (night) at } 9 \text { months not associated with breastfeeding } \\
\text { duration in term infants }\end{array}$ \\
\hline Quillin and Glenn (2004) & USA & CS & Feeding method & $\begin{array}{l}33 \\
1 \text { month }\end{array}$ & $\mathrm{D}$ & $\begin{array}{l}\text { SD ( } 24 \mathrm{~h}, \text { night), NW, } \\
\text { SP ( } 24 \mathrm{~h})\end{array}$ & $\begin{array}{l}\downarrow S D(24 \mathrm{~h}) \text { at } 1 \text { month associated with breastfeeding } \\
\text { SD (night), NW and SP }(24 \mathrm{~h}) \text { not associated with feeding } \\
\text { method }\end{array}$ \\
\hline Ball (2003) & UK & $\mathrm{L}$ & Feeding method & $\begin{array}{l}253 \\
1,3 \text { months }\end{array}$ & $\mathrm{D}$ & NW & $\uparrow \mathrm{NW}$ at 1 and 3 months associated with breastfeeding \\
\hline Lee $(2000)$ & Korea & CS & Feeding method & $\begin{array}{l}188 \\
0-4 \text { months }\end{array}$ & D & SD (24 h), LSP (night) & $\begin{array}{l}\uparrow S D(24 \mathrm{~h}) \text { and } \downarrow \text { LSP (night) at } 0-4 \text { months associated with } \\
\text { breastfeeding }\end{array}$ \\
\hline Thomas (2000) & USA & CS & Feeding method & $\begin{array}{l}37 \\
1 \text { month }\end{array}$ & $\mathrm{D}$ & $\begin{array}{l}\text { SD ( } 24 \text { h, day, night), } \\
\text { WASO }\end{array}$ & $\begin{array}{l}\text { SD ( } 24 \mathrm{~h} \text {, day, night), and WASO not associated with feeding } \\
\text { method in preterm infants }\end{array}$ \\
\hline $\begin{array}{l}\text { Wolke, Söhne, Riegel, Ohrt, and } \\
\text { Österlund (1998) }\end{array}$ & $\begin{array}{l}\text { Finland/ } \\
\text { Germany }\end{array}$ & $\mathrm{L}$ & Feeding method & $\begin{array}{l}5969 \\
5 \text { months }\end{array}$ & I & NW & $\uparrow N W$ at 5 months associated with breastfeeding \\
\hline Quillin (1997) & USA & CS & Feeding method & $\begin{array}{l}44 \\
1 \text { month }\end{array}$ & D & $\begin{array}{l}\text { SD (night), NW, SP } \\
(24 \mathrm{~h})\end{array}$ & $\begin{array}{l}\downarrow \text { SD (night) and } \uparrow \text { NW at } 1 \text { month in breastfed infants } \\
\text { SP ( } 24 \text { h) at } 1 \text { month not associated with feeding method }\end{array}$ \\
\hline Brown and Harries (2015) & UK & CS & Diet & $\begin{array}{l}715 \\
6-12 \text { months }\end{array}$ & Q & NW & $\begin{array}{l}\text { NW at 6-12 months not associated with milk feeds, age of } \\
\text { introduction of solids, number of complementary meals } \\
\text { per day, and daytime feeds. }\end{array}$ \\
\hline Nevarez et al. (2010) & USA & $\mathrm{L}$ & Diet & $\begin{array}{l}1676 \\
6 \text {, and } 12 \text { months }\end{array}$ & Q & SD (24 h) & $\begin{array}{l}\downarrow \mathrm{SD}(24 \mathrm{~h}) \text { at } 12 \text { months associated with introduction of } \\
\text { solids }<4 \text { months }\end{array}$ \\
\hline Thomas and Foreman (2005) & USA & CS & Diet & $\begin{array}{l}37 \\
1-2 \text { months }\end{array}$ & $\mathrm{D}$ & $\begin{array}{l}\text { SD }(24 \mathrm{~h}), \text { SP }(24 \mathrm{~h}) \\
\quad \text { LSP }(24 \mathrm{~h})\end{array}$ & $\begin{array}{l}\downarrow \text { SD }(24 h), \uparrow S P(24 h) \text {, and } \downarrow \text { SD }(24 h) \text { associated with } \uparrow \\
\text { infant feedings }\end{array}$ \\
\hline $\begin{array}{l}\text { Telliez, Bach, Leke, Chardon, and } \\
\text { Libert (2002) }\end{array}$ & France & CS & Diet & $\begin{array}{l}17 \\
1 \text { month }\end{array}$ & $P$ & SD (day) & $\uparrow S D$ (day) in the medium-chain triacylglycerol diet group \\
\hline Philbrook and Teti (2016) & USA & $\mathrm{L}$ & Bedtime routines & $\begin{array}{l}109 \\
1,3 \text { and } 6 \\
\text { months }\end{array}$ & V & SD (night) & $\begin{array}{l}\downarrow S D \text { (night) from } 1 \text { to } 6 \text { months associated with } \downarrow \text { arousing } \\
\text { activities }\end{array}$ \\
\hline
\end{tabular}


Vijakkhana, Wilaisakditipakorn, Thailand Ruedeekhajorn, Pruksananonda,

and Chonchaiya (2015)

Cespedes et al. (2014)

Nevarez et al. (2010)

Mindell et al. (2009)

L Bedtime routines 208

Bedtime routines

L Bedtime routine

CS Bedtime routines

1864

6, 12 months

6 and 12 months

1473

0-11 months

\begin{tabular}{|c|c|c|c|c|}
\hline Philbrook and Teti (2016) & USA & L & $\begin{array}{l}\text { Parental bedtime } \\
\text { involvement }\end{array}$ & $\begin{array}{l}109 \\
1,3 \text { and } 6 \\
\text { months }\end{array}$ \\
\hline Ramamurthy et al. (2012) & $\begin{array}{l}\text { Asia Pacific } \\
\text { Region }\end{array}$ & CS & $\begin{array}{l}\text { Parental bedtime } \\
\text { involvement }\end{array}$ & $\begin{array}{l}4602 \\
0-12 \text { months }\end{array}$ \\
\hline Mindell et al. (2009) & USA & CS & $\begin{array}{l}\text { Parental bedtime } \\
\text { involvement }\end{array}$ & $\begin{array}{l}1473 \\
0-11 \text { months }\end{array}$ \\
\hline Tikotzky and Sadeh (2009) & Israel & L & $\begin{array}{l}\text { Parental bedtime } \\
\text { involvement }\end{array}$ & $\begin{array}{l}85 \\
1,6 \text { and } 12 \\
\text { months }\end{array}$ \\
\hline Anuntaseree et al. (2008) & Thailand & CS & $\begin{array}{l}\text { Parental bedtime } \\
\text { involvement }\end{array}$ & $\begin{array}{l}3172 \\
3 \text { months }\end{array}$ \\
\hline DeLeon and Karraker (2007) & USA & CS & $\begin{array}{l}\text { Parental bedtime } \\
\text { involvement }\end{array}$ & $\begin{array}{l}41 \\
9 \text { months }\end{array}$ \\
\hline Touchette et al. (2005) & Canada & L & $\begin{array}{l}\text { Parental bedtime } \\
\text { involvement }\end{array}$ & $\begin{array}{l}1741 \\
5 \text { months }\end{array}$ \\
\hline Goodlin-Jones et al. (2001) & USA & CS & $\begin{array}{l}\text { Parental bedtime } \\
\text { involvement }\end{array}$ & $\begin{array}{l}80 \\
3,6,9, \text { or } 12 \\
\text { months }\end{array}$ \\
\hline Philbrook and Teti (2016) & USA & L & $\begin{array}{l}\text { Sleep } \\
\text { arrangements }\end{array}$ & $\begin{array}{l}109 \\
1,3 \text { and } 6 \\
\text { months }\end{array}$ \\
\hline Volkovich et al. (2015) & Israel & L & $\begin{array}{l}\text { Sleep } \\
\quad \text { arrangements }\end{array}$ & $\begin{array}{l}153 \\
3 \text { and } 6 \text { months }\end{array}$ \\
\hline
\end{tabular}

SD (night)

SD (24 h)

SD (24 h)

SD (night), NW, SL (night)

V

SD (night)

SD (24 h, day, night),

NW, SP (day), SL (night), LSP (night) BT, WT

SD (night), NW, SL (night)

Combined measures: $A, D$ and $Q$

NW

NW

D

SD (24 h, day, night) WASO, NW, SP (day)

Q SD (night)

V NW, LSP (night)

V SD (night) $\downarrow$ SD (night) at 12 months associated with $\uparrow$ evening media exposure at 6 and 12 months

$\downarrow \mathrm{SD}(24 \mathrm{~h})$ at 6 and 12 months associated with $\uparrow \mathrm{TV}$ viewing

$\downarrow \mathrm{SD}(24 \mathrm{~h})$ at 12 months associated with

infant TV/video viewing

$\downarrow$ SD (night) at 0-11 months associated with no bedtime routine, late bedtimes, and no reading at bedtime

$\uparrow \mathrm{NW}$ at $0-11$ months associated with late bedtimes, and no reading at bedtime but not with no bedtime routine

$\uparrow S L$ (night) at $0-11$ months associated with late bedtimes, but not with no bedtime routine and reading at bedtime $\downarrow$ SD (night) from 1 to 6 months associated with close contact with the mother at bedtime, $\downarrow$ mother emotional availability, $\downarrow$ nursing at bedtime

$\uparrow$ NW and $\downarrow$ LSP in infants fed back to sleep

SD (24 h, day, night), SP (day), SL (night), BT, and WT not associated with fed back to sleep

$\downarrow$ SD (night) and $\uparrow$ NW at 0-11 months associated with put in crib asleep, parent present at bedtime

$\uparrow \mathrm{SL}$ (night) at 0-11 months associated with put in crib asleep

$\uparrow$ NW (measured by A, D, Q) at 6 and 12 months associated with maternal cognitions at pregnancy, 6 and 12 months emphasizing the infant's distress and need for help at night, and $\uparrow$ parental involvement at bedtime at 6 months

$\uparrow N W$ at 3 months associated with use of a swinging or rocking cradle, falling asleep while feeding

$\downarrow$ SD (night) and $\uparrow$ WASO at 9 months associated with $\downarrow$ SD (night) and $\uparrow$ WASO at 9 mon
putting the infant to bed asleep

SD (24 h, day), NW, SP (day) not associated with putting the infant to bed asleep

$\downarrow$ SD (night) at 5 months associated with feeding after awakenings, rocking to sleep, and bought to parent's bed after awakenings

$\uparrow N W$ and $\downarrow$ LSP (night) associated with non-self-soothing style

$\downarrow$ SD (night) from 1 to 6 months associated with co-sleeping

Combined SD (day, night), NW, $\uparrow$ NW (measured by D) associated with co-sleeping infants at measures: A and LSP (night)
3 and 6 months

SD (night), NW, LSP (night) (measured by A), and SD (day) (measured by D) not associated with sleep arrangements 


\begin{tabular}{|c|c|c|c|c|c|c|c|}
\hline First author Year of publication & Country & Design & Associated factor & $\begin{array}{c}N \\
\text { Age } \\
\text { (months) }\end{array}$ & Sleep measure & Sleep-wake behaviour & Main result \\
\hline Huang et al. (2016) & China & $\mathrm{L}$ & $\begin{array}{l}\text { Sleep } \\
\text { arrangements }\end{array}$ & $\begin{array}{l}524 \\
1,3,5, \text { and } 8 \\
\text { months }\end{array}$ & $\mathrm{D}$ & $\begin{array}{l}\text { SD (24 h, day, night), } \\
\text { NW, SP (day), LSP } \\
\text { (night) }\end{array}$ & $\begin{array}{l}\uparrow \mathrm{SD}(24 \mathrm{~h} \text {, day) in the first } 8 \text { months associated with room- } \\
\text { sharing } \\
\uparrow \mathrm{SD} \text { (night) in the first } 8 \text { months associated with } \\
\text { independent sleeping } \\
\text { NW, SP (day), LSP (night) not associated with sleep } \\
\text { arrangements }\end{array}$ \\
\hline Bruni et al. (2014) & Italy & L & $\begin{array}{l}\text { Sleep } \\
\text { arrangements }\end{array}$ & $\begin{array}{l}704 \\
1,3,6,9 \text {, and } 12 \\
\text { months }\end{array}$ & 1 & $\begin{array}{l}\text { SD ( } 24 \text { h, day, night), } \\
\text { NW }\end{array}$ & $\begin{array}{l}\uparrow \mathrm{NW} \text { at } 3,6,9 \text {, and } 12 \text { months associated with bed-sharing } \\
\uparrow \mathrm{SD} \text { (night) at } 3 \text { months, } \uparrow \mathrm{SD}(24 \mathrm{~h} \text {, day) at } 3 \text { and } 6 \text { months, } \\
\text { and } \uparrow \mathrm{SD} \text { (day) at } 12 \text { months in infants associated with } \\
\text { independent sleeping }\end{array}$ \\
\hline $\begin{array}{l}\text { Möllborg, Wennergren, Norvenius, } \\
\text { and Alm (2011) }\end{array}$ & Sweden & CS & $\begin{array}{l}\text { Sleep } \\
\text { arrangements }\end{array}$ & $\begin{array}{l}5605 \\
6 \text { months }\end{array}$ & Q & NW & $\uparrow N W$ at 6 months associated with bed sharing \\
\hline Santos et al. (2008) & Brazil & L & $\begin{array}{l}\text { Sleep } \\
\text { arrangements }\end{array}$ & $\begin{array}{l}3907 \\
0 \text { and } 12 \text { months }\end{array}$ & 1 & NW & $\uparrow \mathrm{NW}$ at 12 months associated with co-sleeping \\
\hline DeLeon and Karraker (2007) & USA & CS & $\begin{array}{l}\text { Sleep } \\
\text { arrangements }\end{array}$ & $\begin{array}{l}41 \\
9 \text { months }\end{array}$ & D & $\begin{array}{l}\text { SD (24 h, day, night), } \\
\text { WASO, NW, SP (day) }\end{array}$ & $\begin{array}{l}\downarrow \mathrm{SD} \text { (night), } \uparrow \text { WASO and } \uparrow \text { NW at } 9 \text { months associated with } \\
\text { co-sleeping } \\
\text { SD ( } 24 \mathrm{~h} \text {, day), and SP (day) not associated with sleep } \\
\text { arrangements }\end{array}$ \\
\hline $\begin{array}{l}\text { Baddock, Galland, Bolton, } \\
\text { Williams, and Taylor (2006) }\end{array}$ & New Zealand & CS & $\begin{array}{l}\text { Sleep } \\
\text { arrangements }\end{array}$ & $\begin{array}{l}80 \\
0-6 \text { months }\end{array}$ & V & SD (night) & $\begin{array}{l}\text { SD (night) at 0-6 months not associated with sleep } \\
\text { arrangements }\end{array}$ \\
\hline Touchette et al. (2005) & Canada & L & $\begin{array}{l}\text { Sleep } \\
\text { arrangements }\end{array}$ & $\begin{array}{l}1741 \\
5 \text { months }\end{array}$ & Q & SD (night) & $\downarrow$ SD (night) at 5 months associated with co-sleeping \\
\hline $\begin{array}{l}\text { Jenni, Fuhrer, Iglowstein, Molinari, } \\
\text { and Largo (2005) }\end{array}$ & Switzerland & L & $\begin{array}{l}\text { Sleep } \\
\text { arrangements }\end{array}$ & $\begin{array}{l}493 \\
1,3,6,9,12 \\
\text { months }\end{array}$ & 1 & SD (night), NW & $\begin{array}{l}\downarrow S D \text { (night) and } \uparrow N W \text { at } 6,9 \text {, and } 12 \text { months associated with } \\
\text { bed sharing }\end{array}$ \\
\hline $\begin{array}{l}\text { Mao, Burnham, Goodlin-Jones, } \\
\text { Gaylor, and Anders (2004) }\end{array}$ & USA & CS & $\begin{array}{l}\text { Sleep } \\
\text { arrangements }\end{array}$ & $\begin{array}{l}18 \\
3,6,9,12 \\
\text { months }\end{array}$ & V & NW & $\uparrow \mathrm{NW}$ at 6,9 , and 12 months associated with co-sleeping \\
\hline $\begin{array}{l}\text { Lukowski, Liu, Peirano, Odio, and } \\
\text { Bauer (2015) }\end{array}$ & China & CS & $\begin{array}{l}\text { Disposable diaper } \\
\text { use }\end{array}$ & $\begin{array}{l}82 \\
6 \text { months }\end{array}$ & D & NW & $\uparrow N W$ at 6 months in infants associated with cloth diapers use \\
\hline Thomas and Spieker (2016) & USA & CS & $\begin{array}{l}\text { Parental mental } \\
\text { health }\end{array}$ & $\begin{array}{l}46 \\
8 \text { months }\end{array}$ & Q & SD (24 h) & $\begin{array}{l}\text { SD }(24 \mathrm{~h}) \text { at } 8 \text { months not associated with maternal fatigue or } \\
\text { depression. }\end{array}$ \\
\hline $\begin{array}{l}\text { Sorondo and Reeb-Sutherland } \\
\text { (2015) }\end{array}$ & USA & $\mathrm{L}$ & $\begin{array}{l}\text { Parental mental } \\
\text { health }\end{array}$ & $\begin{array}{l}40 \\
5,9 \text {, and } 12 \\
\text { months }\end{array}$ & Q & $\begin{array}{l}\text { SD (day, night), } \\
\text { WASO, NW, SL } \\
\text { (night) }\end{array}$ & $\begin{array}{l}\uparrow \mathrm{SD} \text { (day) at } 5 \text { months and } \downarrow \text { SD (day) at } 9 \text { and } 12 \text { months } \\
\text { associated with } \uparrow \text { maternal stress at } 5 \text { months } \\
\text { SD (night), WASO, NW, and SL (night) at } 5,9 \text {, and } 12 \text { months } \\
\text { not associated with maternal stress at } 5 \text { months }\end{array}$ \\
\hline Hughes et al. (2015) & Ireland & CS & $\begin{array}{l}\text { Parental mental } \\
\text { health }\end{array}$ & $\begin{array}{l}11134 \\
9 \text { months }\end{array}$ & Q & SD (night), NW & $\begin{array}{l}\downarrow S D \text { (night) and } \uparrow N W \text { at } 9 \text { months associated with } \uparrow \\
\text { maternal stress and depressive symptoms and } \downarrow \text { self- } \\
\text { reported health }\end{array}$ \\
\hline $\begin{array}{l}\text { Loutzenhiser, McAuslan, and } \\
\text { Sharpe (2015) }\end{array}$ & Canada & L & $\begin{array}{l}\text { Parental mental } \\
\text { health }\end{array}$ & $\begin{array}{l}108 \\
1,3,6 \text { months }\end{array}$ & D & SD (night) & $\downarrow$ SD (night) at 1 month associated with $\uparrow$ maternal fatigue \\
\hline
\end{tabular}


Brand, Furlano, Sidler, Schulz, and Switzerland Holsboer-Trachsler (2014)
L Parental mental 24 health
2 and 3 months

Combined

measures:
Piteo et al. (2013)

Goldberg et al. (2013)

Gress-Smith, Luecken, LemeryChalfant, and Howe (2012)

Sinai and Tikotzky (2012)

Mao, Zhu, and Su (2011)

Nevarez et al. (2010)

Armitage et al. (2009)

Baird, Hill, Kendrick, and Inskip (2009)

Karraker and Young (2007)

O'Connor et al. (2007)
Israel

Australia

China

UK

Warren, Howe, Simmens, and Dahl USA (2006)

Dennis and Ross (2005)

Canada

L Parental mental

$$
\text { health }
$$

L Parental mental

health

6 months
505

6 months

1-2 months

CS Parental sleep

46
46
8 months
Q

SD (day, night), NW

NW, WASO

NW

SD (day), WASO

(night), NW

SD (24 h)

SD (24 h)

6 , and 12 months 18

0 and 6 months

874

6 and 12 months

6 month

11490

6 months NW, SP (day), SL (night)

NW
SD (day, night), NW, SP (day)
NW

NW, LSP (day)

SD (24 h)
NW, SP (day) and $\downarrow$ SD (day) at 2 months associated with $\uparrow$ family strain at 3 months and maternal depression symptoms at 2 and 3 months

$\downarrow \mathrm{SD}$ (night) at 2 months associated with $\uparrow$ maternal depression symptoms at 2 months

$\downarrow$ SD (night) at 3 months associated with $\uparrow$ maternal depression symptoms at 3 months

$\downarrow \mathrm{SD}$ (day, night) and $\uparrow \mathrm{NW}$ at 12 months associated with $\uparrow$ family dysfunction

$\downarrow \mathrm{SD}$ (night) and $\uparrow \mathrm{NW}$ at 12 months associated with $\uparrow$ postnatal depression

$\uparrow \mathrm{NW}$ and $\uparrow$ WASO at 6 and 12 months associated with $\uparrow$ maternal depressive symptoms at 6 and 12 months

$\uparrow \mathrm{NW}$ at 9 months associated with $\uparrow$ maternal depressive symptoms at 5 months

$\downarrow$ SD (day), $\uparrow$ WASO associated with $\uparrow$ parenting stress in families whose mothers in maternity leave

NW not associated with parenting stress in families whose mothers in maternity leave

$\downarrow$ SD $(24 \mathrm{~h})$ at $1-2$ months associated with $\uparrow$ maternal postpartum depression

$\downarrow \mathrm{SD}(24 \mathrm{~h})$ at 12 months associated with $\uparrow$ maternal antenatal depression

S (24 h, day, night), $\downarrow$ SD (night), $\uparrow$ SP (day), $\uparrow$ NW, $\uparrow$ SL (night) at 0 and 6 months

in associated with maternal history of depression

SD ( $24 \mathrm{~h}$, day) not associated with maternal history of depression

NW at 6 and 12 months associated with maternal preconception psychological distress

$\uparrow \mathrm{NW}$ at 6 months associated with $\uparrow$ maternal depression symptoms at 6 months

SD $(24 \mathrm{~h})$, and NW at 6 months not associated with prenatal maternal anxiety and depression

$\downarrow$ SD (24 h) associated with maternal depression at 8 weeks and 8 months and anxiety at 8 weeks

$\uparrow$ NW associated with maternal depression and anxiety at 8 months

$\uparrow N W$ at 6 months associated with $\uparrow$ maternal depression symptoms at 1, 6, 15, 24 and 36 months

$\uparrow \mathrm{NW}$ at 1 and 2 months associated with $\uparrow$ maternal postpartum depression at 1 and 2 months LSP (day) at 1 and 2 months not associated with maternal postpartum depression at 1 and 2 months

$\downarrow$ SD $(24 \mathrm{~h})$ at 8 months moderately associated with $\downarrow$ maternal sleep and $\uparrow$ maternal wake disturbance 


\begin{tabular}{|c|c|c|c|c|c|c|c|}
\hline First author Year of publication & Country & Design & Associated factor & $\begin{array}{c}N \\
\text { Age } \\
\text { (months) }\end{array}$ & Sleep measure & Sleep-wake behaviour & Main result \\
\hline $\begin{array}{l}\text { Sharkey, Iko, Machan, Thompson- } \\
\text { Westra, and Pearlstein (2016) }\end{array}$ & USA & $\mathrm{L}$ & Parental sleep & $\begin{array}{l}31 \\
0,1, \text { and } 4 \\
\text { months }\end{array}$ & $\mathrm{D}$ & $\begin{array}{l}\text { SD (24 h), SP (24 h), } \\
\text { LSP (24 h), BT }\end{array}$ & $\begin{array}{l}\downarrow \text { LSP }(24 \mathrm{~h}) \text { at } 0 \text { months associated with } \downarrow \text { maternal SD and } \downarrow \\
\text { maternal SE at } 0 \text { months } \\
\downarrow \text { SP }(24 \mathrm{~h}) \text { at } 0 \text { months associated with } \uparrow \text { maternal SE at } 0 \\
\text { months } \\
\text { SD }(24 \mathrm{~h}), \text { SP ( } 24 \mathrm{~h}), \text { LSP ( } 24 \mathrm{~h}), \mathrm{BT} \text { at } 1 \text { and } 4 \text { months not } \\
\text { associated with maternal sleep }\end{array}$ \\
\hline Tikotzky et al. (2015) & Israel & $\mathrm{L}$ & Parental sleep & $\begin{array}{l}57 \\
3 \text { and } 6 \text { months }\end{array}$ & $\begin{array}{l}\text { Combined } \\
\text { measures: A, D, } \\
\text { and Q }\end{array}$ & NW, SE & $\begin{array}{l}\uparrow N W \text { and } \uparrow S E \text { at } 3 \text { and } 6 \text { months associated with } \uparrow \text { maternal } \\
N W \text { and } \uparrow S E \text { at } 3 \text { and } 6 \text { months }\end{array}$ \\
\hline Brand et al. (2014) & Switzerland & $\mathrm{L}$ & Parental sleep & $\begin{array}{l}24 \\
2 \text { and } 3 \text { months }\end{array}$ & $\begin{array}{l}\text { Combined } \\
\text { measures: A and } \\
\text { D }\end{array}$ & $\begin{array}{l}\text { SD (day, night), NW, } \\
\text { SP (day) }\end{array}$ & $\begin{array}{l}\uparrow N W \text { and SP (day) and } \downarrow \text { SD (day) at } 2 \text { months associated } \\
\text { with } \uparrow \text { maternal insomnia at } 2 \text { and } 3 \text { months } \\
\downarrow \text { SD (night) at } 2 \text { months associated with } \uparrow \text { maternal } \\
\text { insomnia at } 2 \text { months } \\
\uparrow S P \text { (day) at } 3 \text { months associated with } \uparrow \text { maternal insomnia } \\
\text { at } 3 \text { months }\end{array}$ \\
\hline Demirci et al. (2012) & USA & CS & Parental sleep & $\begin{array}{l}77 \\
6-11 \text { months }\end{array}$ & Q & SD (night) & $\downarrow$ SD (night) at 6-11 months associated with $\downarrow$ maternal SD \\
\hline Sinai and Tikotzky (2012) & Israel & CS & Parental sleep & $\begin{array}{l}50 \\
4-5 \text { months }\end{array}$ & $\mathrm{D}$ & SD (day), WASO, NW & $\begin{array}{l}\uparrow N W \text { and } \uparrow N A \text { associated with } \uparrow N W \text { and } \uparrow \text { WASO of } \\
\text { mothers and fathers } \\
\text { SD (day) at 4-5 months not associated with parental sleep }\end{array}$ \\
\hline Thomas and Foreman (2005) & USA & CS & Parental sleep & $\begin{array}{l}37 \\
1-2 \text { months }\end{array}$ & $\mathrm{D}$ & $\begin{array}{l}\text { SD }(24 h) \\
\operatorname{LSP}(24 h)\end{array}$ & $\begin{array}{l}\downarrow \text { LSP }(24 \mathrm{~h}) \text { associated with } \downarrow \text { maternal LSP }(24 \mathrm{~h}) \text { and } \downarrow \text { SP } \\
(24 \mathrm{~h}) \\
\text { SD }(24 \mathrm{~h}) \text { at } 1-2 \text { months not associated with maternal sleep }\end{array}$ \\
\hline Tikotzky et al. (2015) & Israel & $\mathrm{L}$ & $\begin{array}{l}\text { Parental } \\
\text { involvement }\end{array}$ & $\begin{array}{l}57 \\
3 \text { and } 6 \text { months }\end{array}$ & $\begin{array}{l}\text { Combined } \\
\text { measures: A, D, } \\
\text { and Q }\end{array}$ & SD (night), NW, SE & $\begin{array}{l}\uparrow \text { NW at } 6 \text { months (measured by A and D) associated with } \downarrow \\
\text { involvement of fathers in infant care at } 3 \text { and } 6 \text { months } \\
\uparrow N W \text { at } 3 \text { months (measured by D) associated with } \downarrow \\
\text { involvement of fathers in infant care at } 6 \text { months } \\
\text { SD (night) and SE at } 3 \text { and } 6 \text { months not associated with } \\
\text { parental involvement }\end{array}$ \\
\hline Zaidman-Zait and Hall (2015) & Canada & $\mathrm{L}$ & $\begin{array}{l}\text { Parental } \\
\quad \text { involvement }\end{array}$ & $\begin{array}{l}1487 \\
5 \text { months }\end{array}$ & Q & NW & $\begin{array}{l}\uparrow \mathrm{NW} \text { at } 5 \text { months associated with } \downarrow \text { maternal parenting } \\
\text { impact and self-efficacy scores and } \downarrow \text { paternal parenting } \\
\text { impact and } \uparrow \text { overprotectiveness scores }\end{array}$ \\
\hline $\begin{array}{l}\text { Bordeleau, Bernier, and Carrier } \\
\text { (2012) }\end{array}$ & Canada & $\mathrm{L}$ & $\begin{array}{l}\text { Parental } \\
\text { involvement }\end{array}$ & $\begin{array}{l}55 \\
12 \text { months }\end{array}$ & D & SD (24 h, night) & $\begin{array}{l}\text { SD ( } 24 \mathrm{~h}, \text { night) at } 12 \text { months not associated with maternal } \\
\text { sensitivity at } 12 \text { months }\end{array}$ \\
\hline Tikotzky, Sadeh, et al. (2010) & Israel & $\mathrm{L}$ & $\begin{array}{l}\text { Parental } \\
\text { involvement }\end{array}$ & $\begin{array}{l}56 \\
1 \text { and } 6 \text { months }\end{array}$ & $\begin{array}{l}\text { Combined } \\
\text { measures: A and } \\
\text { D }\end{array}$ & SD (night), NW, BT & $\begin{array}{l}\downarrow \text { SD (night) and } \downarrow \text { NW at } 6 \text { months associated with } \uparrow \\
\text { paternal involvement in overall infant care at } 1 \text { and } 6 \\
\text { months } \\
\uparrow \text { BT at } 6 \text { months associated with } \uparrow \text { paternal involvement in } \\
\text { overall infant care at } 6 \text { months, but not at } 1 \text { month }\end{array}$ \\
\hline Scher and Blumberg (1999) & Israel & $\mathrm{L}$ & $\begin{array}{l}\text { Parental } \\
\text { involvement }\end{array}$ & $\begin{array}{l}81 \\
6 \text { and } 12 \text { months }\end{array}$ & Q & NW & $\begin{array}{l}\uparrow \mathrm{NW} \text { at } 12 \text { months associated with a facilitator care-giving } \\
\text { orientation }\end{array}$ \\
\hline
\end{tabular}




\begin{tabular}{|c|c|c|c|c|c|c|c|}
\hline Atun-Einy and Scher (2016) & Israel & L & $\begin{array}{l}\text { Motor } \\
\text { development }\end{array}$ & $\begin{array}{l}20 \\
7,8,9,10,11 \\
\text { months }\end{array}$ & A & NW, SE & $\begin{array}{l}\text { NW not associated with the emergence of pulling-to-stand } \\
\uparrow \mathrm{NW} \text { and } \downarrow \mathrm{SE} \text { associated with crawling onset in infants with } \\
\text { early crawling }\end{array}$ \\
\hline Mindell and Lee (2015) & Brazil & CS & $\begin{array}{l}\text { Motor } \\
\text { development }\end{array}$ & $\begin{array}{l}1351 \\
3-13 \text { months }\end{array}$ & Q & $\begin{array}{l}\text { SD (night), SL (night), } \\
\text { NW, SP (day), BT, } \\
\text { WT }\end{array}$ & $\begin{array}{l}\text { SD (night), SL (night), NW, SP (day), BT, and WT at 3-13 } \\
\text { months not associated with fine and gross motor skills } \\
\text { scores }\end{array}$ \\
\hline Scher and Cohen (2015) & Israel & L & $\begin{array}{l}\text { Motor } \\
\text { development }\end{array}$ & $\begin{array}{l}28 \\
5,6,7,8,9,10 \\
\text { and } 11 \text { months }\end{array}$ & A & NW & $\begin{array}{l}\uparrow N W \text { associated with crawling onset in infants with early } \\
\text { crawling }\end{array}$ \\
\hline Gibson, Elder, and Gander (2012) & New Zealand & CS & $\begin{array}{l}\text { Motor } \\
\text { development }\end{array}$ & $\begin{array}{l}52 \\
12 \text { months }\end{array}$ & A & SD (24 h, night), SE & $\begin{array}{l}\text { SD ( } 24 \mathrm{~h} \text {, night) and SE at } 12 \text { months not associated with } \\
\text { motor development }\end{array}$ \\
\hline Scher (2005a) & Israel & CS & $\begin{array}{l}\text { Motor } \\
\text { development }\end{array}$ & $\begin{array}{l}59 \\
8 \text { months }\end{array}$ & $\begin{array}{l}\text { Combined } \\
\text { measures: } A \text { and } \\
\text { Q }\end{array}$ & $\begin{array}{l}\text { SD (night), NW, LSP } \\
\text { (night), SE, BT }\end{array}$ & $\begin{array}{l}\uparrow \mathrm{NW} \text { at } 8 \text { months associated with crawling } \\
\text { SD (night), LSP (night), SE, and BT at } 8 \text { months not associated } \\
\text { with motor development }\end{array}$ \\
\hline Scher and Cohen (2005) & Israel & CS & $\begin{array}{l}\text { Motor } \\
\text { development }\end{array}$ & $\begin{array}{l}107 \\
5-8 \text { months }\end{array}$ & Q & NW & $\uparrow N W$ at 5-8 months associated with crawling \\
\hline Küpers et al. (2015) & Netherlands & L & Physical growth & $\begin{array}{l}2475 \\
1,6, \text { and } 12 \\
\text { months }\end{array}$ & NS & SD (24 h) & $\uparrow \mathrm{SD}(24 \mathrm{~h})$ at 4 months associated with $\downarrow$ weight gain for age \\
\hline $\begin{array}{l}\text { Hiscock, Scalzo, Canterford, and } \\
\text { Wake (2011) }\end{array}$ & Australia & L & Physical growth & $\begin{array}{l}3857 \\
0-12 \text { months }\end{array}$ & D & SD $(24 \mathrm{~h})$ & $\begin{array}{l}\text { SD }(24 \mathrm{~h}) \text { at } 0-12 \text { months not associated with body mass } \\
\text { index at } 0-12 \text { months and later }\end{array}$ \\
\hline Lampl and Johnson (2011) & USA & L & Physical growth & $\begin{array}{l}23 \\
0-12 \text { months }\end{array}$ & D & $\begin{array}{l}\text { SD ( } 24 \mathrm{~h}), \text { SP }(24 \mathrm{~h}) \\
\quad \operatorname{LSP}(24 \mathrm{~h})\end{array}$ & $\begin{array}{l}\uparrow S D(24 h) \text { and SP }(24 h) \text { in the first } 12 \text { months associated } \\
\text { with episodic growth in length } \\
\text { LSP }(24 \mathrm{~h}) \text { not associated with episodic growth in length }\end{array}$ \\
\hline Tikotzky, De Marcas, et al. (2010) & Israel & CS & Physical growth & $\begin{array}{l}96 \\
6 \text { months }\end{array}$ & $\begin{array}{l}\text { Combined } \\
\text { measures: A and } \\
\text { Q }\end{array}$ & $\begin{array}{l}\text { SD (day, night), NW, } \\
\text { SE }\end{array}$ & $\begin{array}{l}\downarrow \text { SD (night) (measured by Q) and } \downarrow \text { SE at } 6 \text { months } \\
\text { associated with } \uparrow \text { weight-to-length ratio and } \uparrow \text { weight } \\
\text { above weight expected for length } \\
\uparrow \text { SE at } 6 \text { months associated with } \uparrow \text { length } \\
\text { NW (measured by A and Q), SD (night) (measured by A), and } \\
\text { SD (day) (measured by Q) at } 6 \text { months not associated with } \\
\text { physical growth }\end{array}$ \\
\hline Kirjavainen et al. (2001) & Finland & $\mathrm{L}$ & Colic & $\begin{array}{l}31 \\
1,2,6,7 \text { months }\end{array}$ & $\begin{array}{c}\text { Combined } \\
\text { measures: } \mathrm{D} \text { and } P\end{array}$ & SD (24 h, night), NW & $\begin{array}{l}\downarrow S D(24 \mathrm{~h} \text {, night) at } 1 \text { month in infants with colic } \\
\downarrow \mathrm{NW} \text { at } 7 \text { months in infants with colic } \\
\text { SD ( } 24 \mathrm{~h} \text {, night) at } 2,6 \text {, and } 7 \text { months not associated with } \\
\text { colic }\end{array}$ \\
\hline $\begin{array}{l}\text { White, Gunnar, Larson, Donzella, } \\
\text { and Barr (2000) }\end{array}$ & USA & CS & Colic & $\begin{array}{l}40 \\
2 \text { months }\end{array}$ & D & SD (24 h, day, night) & $\downarrow \mathrm{SD}(24 \mathrm{~h})$ at 2 months associated with colic \\
\hline Anuntaseree et al. (2012) & Thailand & CS & Health problems & $\begin{array}{l}4085 \\
12 \text { months }\end{array}$ & 1 & $\begin{array}{l}\text { SD (day, night), NW, } \\
\text { SP (day), SL (night) }\end{array}$ & $\begin{array}{l}\downarrow S L \text { (night) and } \downarrow \text { SD (night) at } 12 \text { months in infants } \\
\text { associated with atopic dermatitis } \\
\text { SD (day), NW and SP (day) not associated with atopic } \\
\quad \text { dermatitis }\end{array}$ \\
\hline Pellegrini-Belinchón et al. (2012) & Spain & CS & Health problems & $\begin{array}{l}750 \\
12 \text { months }\end{array}$ & Q & NW & $\uparrow \mathrm{NW}$ at 12 months associated with wheezing \\
\hline Kozyrskyj et al. (2009) & Australia & L & Health problems & $\begin{array}{l}2398 \\
12 \text { months }\end{array}$ & Q & NW & NW at 12 months not associated with asthma at 6 years \\
\hline
\end{tabular}




\begin{tabular}{|c|c|c|c|c|c|c|c|}
\hline First author Year of publication & Country & Design & Associated factor & $\begin{array}{c}N \\
\text { Age } \\
\text { (months) }\end{array}$ & Sleep measure & Sleep-wake behaviour & Main result \\
\hline $\begin{array}{l}\text { McKay and Angulo-Barroso } \\
\text { (2006) }\end{array}$ & USA & $\mathrm{L}$ & Health problems & $\begin{array}{l}16 \\
3,4,5, \text { and } 6 \\
\text { months }\end{array}$ & $\mathrm{A}$ & SD $(24 \mathrm{~h})$ & $\downarrow \mathrm{SD}(24 \mathrm{~h})$ at 3 months associated with Down Syndrome \\
\hline Wolke et al. (1998) & $\begin{array}{l}\text { Finland/ } \\
\text { Germany }\end{array}$ & L & Health problems & $\begin{array}{l}5969 \\
5 \text { months }\end{array}$ & 1 & NW & $\begin{array}{l}\downarrow N W \text { at } 5 \text { months associated with admission to special care } \\
\text { units }\end{array}$ \\
\hline Ghaem et al. (1998) & Australia & CS & Health problems & $\begin{array}{l}102 \\
0-3 \text { and } 3-12 \\
\text { months }\end{array}$ & Q & SD (day), NW & $\begin{array}{l}\uparrow \mathrm{NW} \text { at } 3-12 \text { months associated with gastro-esophageal } \\
\text { reflux disease } \\
\text { SD (day) at 3-12 months not associated with gastro- } \\
\text { esophageal reflux disease }\end{array}$ \\
\hline $\begin{array}{l}\text { Konrad, Herbert, Schneider, and } \\
\text { Seehagen (2016) }\end{array}$ & UK & CS & $\begin{array}{l}\text { Mental } \\
\text { development }\end{array}$ & $\begin{array}{l}48 \\
6 \text { and } 12 \text { months }\end{array}$ & $\begin{array}{l}\text { Combined } \\
\text { measures: } A \text { and } \\
D \text { (combined) }\end{array}$ & $\begin{array}{l}\text { SD (24 h, day, night), } \\
\text { WASO, NW, SP (day) }\end{array}$ & $\begin{array}{l}\uparrow \text { WASO and NW at } 6 \text { months infants (but not at } 12 \text { months) } \\
\text { associated with } \downarrow \text { imitation score } \\
\text { SP (day), SD ( } 24 \text { h, day, night) not associated with imitation } \\
\text { score }\end{array}$ \\
\hline Mindell and Lee (2015) & Brazil & CS & $\begin{array}{l}\text { Mental } \\
\text { development }\end{array}$ & $\begin{array}{l}1351 \\
3-13 \text { months }\end{array}$ & Q & $\begin{array}{l}\text { SD (night), SL (night), } \\
\text { NW. SP (day), BT, } \\
\text { WT }\end{array}$ & $\begin{array}{l}\text { SD (night), SL (night), NW, SP (day), BT, and WT at 3-13 } \\
\text { months not associated with problem solving, } \\
\text { communication, and personal-social skills scores }\end{array}$ \\
\hline Lukowski and Milojevich (2013) & USA & CS & $\begin{array}{l}\text { Mental } \\
\text { development }\end{array}$ & $\begin{array}{l}21 \\
10 \text { months }\end{array}$ & Q & SD (day, night), NW & $\begin{array}{l}\uparrow \text { SD (day) at } 10 \text { months associated with } \uparrow \text { encoding } \\
\downarrow N W \text { and } \uparrow \text { SD (day) at } 10 \text { months associated with } \uparrow \\
\text { generalization } \\
\text { SD (day, night) and NW not associated with delayed recall }\end{array}$ \\
\hline Gibson, Elder, et al. (2012) & New Zealand & CS & $\begin{array}{l}\text { Mental } \\
\text { development }\end{array}$ & $\begin{array}{l}52 \\
12 \text { months }\end{array}$ & A & SE & $\uparrow \mathrm{SE}$ at 12 months associated with $\uparrow$ problem solving \\
\hline Scher (2005b) & Israel & CS & $\begin{array}{l}\text { Mental } \\
\text { development }\end{array}$ & $\begin{array}{l}50 \\
10 \text { months }\end{array}$ & $\begin{array}{l}\text { Combined } \\
\text { measures: A and } \\
\text { Q }\end{array}$ & SD (24 h), NW, SE & $\begin{array}{l}\downarrow N W \text { and } \uparrow \text { SE at } 10 \text { months (measured by A) associated with } \\
\uparrow \text { mental developmental index } \\
\text { SD }(24 \mathrm{~h} \text { ) and NW (measured by Q) not associated with } \\
\text { mental development index }\end{array}$ \\
\hline $\begin{array}{l}\text { Shibagaki, Sawata, and Tachibana } \\
\text { (2004) }\end{array}$ & Japan & CS & $\begin{array}{l}\text { Mental } \\
\text { development }\end{array}$ & $\begin{array}{l}27 \\
4-12 \text { months }\end{array}$ & $P$ & SD (night), WASO & $\begin{array}{l}\downarrow \text { WASO associated with } \uparrow \text { developmental quotient in infants } \\
\text { with developmental disabilities } \\
\text { SD (night) not associated with developmental quotient }\end{array}$ \\
\hline Gertner et al. (2002) & Israel & L & $\begin{array}{l}\text { Mental } \\
\text { development }\end{array}$ & $\begin{array}{l}34 \\
0 \text { and } 6 \text { months }\end{array}$ & A & SD (24 h, day, night) & $\begin{array}{l}\uparrow \text { SD ( } 24 \mathrm{~h} \text {, night) at } 0 \text { months associated with } \downarrow \text { mental } \\
\text { developmental scores at } 6 \text { months } \\
\text { SD (day) at } 0 \text { months not associated with mental } \\
\text { developmental scores at } 6 \text { months }\end{array}$ \\
\hline $\begin{array}{l}\text { McGeorge, Milne, Cotton, and } \\
\text { Whelan (2015) }\end{array}$ & Australia & CS & $\begin{array}{l}\text { Social emotional } \\
\text { problems }\end{array}$ & $\begin{array}{l}55 \\
4-7 \text { months }\end{array}$ & D & SD $(24 \mathrm{~h})$ & $\begin{array}{l}\downarrow \mathrm{SD}(24 \mathrm{~h}) \text { at } 4-7 \text { months associated with } \uparrow \text { sensory } \\
\text { sensitivity and sensation avoiding }\end{array}$ \\
\hline Humphreys et al. (2014) & UK & $\mathrm{L}$ & $\begin{array}{l}\text { Social emotional } \\
\text { problems }\end{array}$ & $\begin{array}{l}14062 \\
6 \text { months }\end{array}$ & Q & SD (24 h), NW & SD (24 h) and NW at 6 months not associated with autism \\
\hline Landau et al. (2010) & Israel & CS & $\begin{array}{l}\text { Social emotional } \\
\text { problems }\end{array}$ & $\begin{array}{l}44 \\
1-2 \text { months }\end{array}$ & $\begin{array}{l}\text { Combined } \\
\text { measures: } A \text { and } \\
\text { D }\end{array}$ & $\begin{array}{l}\text { SD (24 h, day, night), } \\
\text { NW, SP (day) }\end{array}$ & $\begin{array}{l}\text { SD ( } 24 \mathrm{~h} \text {, day, night), NW, and SP (day) not associated with } \\
\text { Attention Deficit Hyperactivity Disorder } \\
\uparrow \text { stability in SD (day) and SP (day) associated with } \downarrow \text { risk for } \\
\text { Attention Deficit Hyperactivity Disorder }\end{array}$ \\
\hline
\end{tabular}




\begin{tabular}{|c|c|c|c|c|c|c|c|}
\hline Scher (2008) & Israel & CS & $\begin{array}{l}\text { Social emotional } \\
\text { problems }\end{array}$ & $\begin{array}{l}52 \\
10 \text { months }\end{array}$ & $\begin{array}{l}\text { Combined } \\
\text { measures: A and } \\
\text { Q }\end{array}$ & NW, SE & $\begin{array}{l}\uparrow \mathrm{NW} \text { (measured by A, but not measured by Q) and } \downarrow \text { SE at } 10 \\
\text { months associated with } \uparrow \text { maternal separation anxiety at } \\
10 \text { months }\end{array}$ \\
\hline Spruyt et al. (2008) & Belgium & $\mathrm{L}$ & $\begin{array}{l}\text { Social emotional } \\
\text { problems }\end{array}$ & $\begin{array}{l}20 \\
3,6,11 \text {, and } 12 \\
\text { months }\end{array}$ & $\begin{array}{l}\text { Combined } \\
\text { measures: A and } \\
\text { D }\end{array}$ & SD (day) & $\begin{array}{l}\uparrow \text { SD (day) at } 12 \text { months associated with } \downarrow \text { emotion } \\
\text { regulation and } \downarrow \text { total behaviour development score }\end{array}$ \\
\hline DeLeon and Karraker (2007) & USA & CS & $\begin{array}{l}\text { Social emotional } \\
\text { problems }\end{array}$ & $\begin{array}{l}41 \\
9 \text { months }\end{array}$ & D & $\begin{array}{l}\text { SD ( } 24 \text { h, day, night), } \\
\text { WASO, NW, SP (day) }\end{array}$ & $\begin{array}{l}\downarrow \mathrm{SD} \text { ( } 24 \mathrm{~h}, \text { night), } \uparrow \text { WASO, } \uparrow \mathrm{NW}, \uparrow \mathrm{SP} \text { (day) at } 9 \text { months } \\
\text { associated with } \uparrow \text { separation distress } \\
\text { SD (night) not associated with separation distress }\end{array}$ \\
\hline Scher and Blumberg (1999) & Israel & L & $\begin{array}{l}\text { Social emotional } \\
\text { problems }\end{array}$ & $\begin{array}{l}81 \\
6 \text { and } 12 \text { months }\end{array}$ & Q & NW & $\begin{array}{l}\uparrow \mathrm{NW} \text { at } 12 \text { months associated with } \uparrow \text { maternal separation } \\
\text { anxiety }\end{array}$ \\
\hline Mindell and Lee (2015) & Brazil & CS & Temperament & $\begin{array}{l}1351 \\
3-13 \text { months }\end{array}$ & Q & $\begin{array}{l}\text { SD (night), SL (night), } \\
\text { NW, SP (day), BT, } \\
\text { WT }\end{array}$ & $\begin{array}{l}\uparrow \text { NW and } \downarrow \text { SP (day) associated with } \downarrow \text { bedtime, morning, } \\
\text { and daytime mood } \\
\uparrow \text { BT and } \downarrow \text { SD (night) associated with } \downarrow \text { daytime mood } \\
\downarrow \text { SD (night) and } \uparrow \text { WT associated with } \downarrow \text { morning mood } \\
\text { SL not associated with mood } \\
\text { BT not associated with bedtime and morning mood } \\
\text { WT not associated with bedtime and daytime mood } \\
\text { SD (night) not associated with bedtime mood }\end{array}$ \\
\hline $\begin{array}{l}\text { Sorondo and Reeb-Sutherland } \\
\text { (2015) }\end{array}$ & USA & L & Temperament & $\begin{array}{l}40 \\
5,9, \text { and } 12 \\
\text { months }\end{array}$ & Q & $\begin{array}{l}\text { SD (day, night), } \\
\text { WASO, NW, SL } \\
\text { (night) }\end{array}$ & $\begin{array}{l}\uparrow S L \text { (night) at } 5 \text { months and } \uparrow \text { WASO at } 5,9 \text {, and } 12 \text { months } \\
\text { associated with negative reactivity at } 5 \text { months } \\
\text { SD (day, night) and NW at } 5,9 \text {, and } 12 \text { months not associated } \\
\text { with negative reactivity }\end{array}$ \\
\hline Kaley et al. (2012) & UK & CS & Temperament & $\begin{array}{l}77 \\
1-2 \text { months }\end{array}$ & D & $\begin{array}{l}\text { SD (24 h, day, night), } \\
\text { NW }\end{array}$ & $\begin{array}{l}\uparrow \mathrm{SD} \text { (day) at } 1-2 \text { months associated with positive } \\
\text { temperament } \\
\downarrow \text { SD ( } 24 \mathrm{~h} \text {, day, night) at } 1-2 \text { months correlated with } \\
\text { temperament dimensions (activity, approach, intensity, } \\
\text { and distractibility) } \\
\text { NW at } 1-2 \text { months not associated with temperament } \\
\text { dimensions }\end{array}$ \\
\hline Gibson, Gander, and Elder (2012) & New Zealand & CS & Temperament & 52 months & A & $\begin{array}{l}\text { SD (night), SL (night), } \\
\text { BT, WT }\end{array}$ & $\begin{array}{l}\downarrow S L \text { (night) at } 12 \text { months in infants more alert during the day } \\
\uparrow \text { SD (night) and } \uparrow \text { WT at } 12 \text { months in infants in good mood } \\
\text { in the morning } \\
\downarrow \text { WT at } 12 \text { months in infants rated as tired at bedtime } \\
\text { SD (night), BT, and WT at } 12 \text { months not associated with } \\
\text { daytime alertness } \\
\text { SL (night) and BT at } 12 \text { months not associated with morning } \\
\text { mood } \\
\text { SD (night), SL (night), and BT at } 12 \text { months not associated } \\
\text { with tired at bedtime } \\
\text { SD (night), SL (night), BT and WT at } 12 \text { months not associated } \\
\text { with bedtime mood and bedtime problems }\end{array}$ \\
\hline Spruyt et al. (2008) & Belgium & L & Temperament & $\begin{array}{l}20 \\
3,6,11 \text {, and } 12 \\
\text { months }\end{array}$ & $\begin{array}{l}\text { Combined } \\
\text { measures: A and } \\
\text { D }\end{array}$ & SD (24 h, day, night) & $\begin{array}{l}\uparrow \mathrm{SD}(24 \mathrm{~h}) \text { at } 3 \text { months associated with } \uparrow \text { approachability } \\
\uparrow \mathrm{SD}(24 \mathrm{~h}) \text { at } 6 \text { months associated with } \uparrow \text { rhythmicity and } \downarrow \\
\text { distractibility } \\
\uparrow \mathrm{SD}(24 \mathrm{~h}) \text { at } 12 \text { months associated with } \uparrow \text { rhythmicity and } \downarrow \\
\text { persistence }\end{array}$ \\
\hline
\end{tabular}




\begin{tabular}{|c|c|c|c|c|c|c|c|}
\hline First author Year of publication & Country & Design & Associated factor & $\begin{array}{c}N \\
\text { Age } \\
\text { (months) }\end{array}$ & Sleep measure & Sleep-wake behaviour & Main result \\
\hline & & & & & & & $\begin{array}{l}\uparrow S D \text { (day) at } 12 \text { months associated with perceived negative } \\
\text { mood } \\
\uparrow S D \text { (night) at } 3,6 \text { and } 11 \text { months associated with } \uparrow \\
\text { approachability }\end{array}$ \\
\hline Palmstierna et al. (2008) & Sweden & L & Temperament & $\begin{array}{l}16467 \\
12 \text { months }\end{array}$ & Q & NW & $\uparrow$ NW at 12 months associated with difficult temperament \\
\hline $\begin{array}{l}\text { Bayer, Hiscock, Hampton, and } \\
\text { Wake (2007) }\end{array}$ & Australia & CS & Temperament & $\begin{array}{l}692 \\
3-6 \text { months }\end{array}$ & Q & NW & $\uparrow N W$ at 3-6 months associated with difficult temperament \\
\hline Touchette et al. (2005) & Canada & L & Temperament & $\begin{array}{l}1741 \\
5 \text { months }\end{array}$ & Q & SD (night) & $\begin{array}{l}\downarrow \text { SD (night) at } 5 \text { months associated with difficult } \\
\text { temperament }\end{array}$ \\
\hline Scher (2001a) & Israel & CS & Temperament & $\begin{array}{l}94 \\
12 \text { months }\end{array}$ & A & $\begin{array}{l}\text { SD (night), NW, LSP } \\
\text { (night), SL (night), } \\
\text { SE }\end{array}$ & $\begin{array}{l}\uparrow \text { SE at } 12 \text { months associated with } \uparrow \text { fussiness } \\
\text { SD (night), NW, LSP (night), and SL (night) at } 12 \text { months not } \\
\text { associated with fussiness }\end{array}$ \\
\hline Scher, Tirosh, and Lavie (1998) & Israel & CS & Temperament & $\begin{array}{l}30 \\
12 \text { months }\end{array}$ & A & SD (night), NW, SE, BT & $\begin{array}{l}\uparrow \mathrm{SD} \text { (night), } \uparrow \mathrm{NW}, \downarrow \mathrm{SE} \text {, and } \downarrow \mathrm{BT} \text { at } 12 \text { months associated } \\
\text { with } \uparrow \text { rhythmicity } \\
\downarrow \mathrm{BT} \text { at } 12 \text { months associated with positive mood } \\
\text { SD, NW, SE, BT at } 12 \text { months not associated with other } \\
\text { dimensions of temperament }\end{array}$ \\
\hline Gibson, Gander, et al. (2012) & New Zealand & CS & Sleep problems & $\begin{array}{l}52 \\
12 \text { months }\end{array}$ & A & $\begin{array}{l}\text { SD ( } 24 \text { h, day, night), } \\
\text { SL (night), SP ( } 24 \text { h), } \\
\text { SE, BT, WT }\end{array}$ & $\begin{array}{l}\downarrow \mathrm{SD}(24 \mathrm{~h} \text {, night) and } \uparrow \mathrm{SP}(24 \mathrm{~h}) \text { at } 12 \text { months in infants } \\
\text { rated with sleep problems } \\
\text { SD (day), SL (night), SE, BT, and WT at } 12 \text { months not } \\
\text { associated with sleep problems }\end{array}$ \\
\hline Bayer et al. (2007) & Australia & CS & Sleep problems & $\begin{array}{l}692 \\
3-6 \text { months }\end{array}$ & Q & NW & $\begin{array}{l}\uparrow N W \text { at 3-6 months associated with maternal report of sleep } \\
\text { problem }\end{array}$ \\
\hline Hiscock and Wake (2001) & Australia & CS & Sleep problems & $\begin{array}{l}738 \\
7-9 \text { months }\end{array}$ & Q & $\begin{array}{l}\text { NW, SP (day), SL, BT, } \\
\text { WT }\end{array}$ & $\begin{array}{l}\uparrow N W, \downarrow S P \text { (day) and } \uparrow S L \text { at } 7-9 \text { months in infants reported } \\
\text { by mothers as presenting a sleep problem } \\
\uparrow \mathrm{BT} \text { and } \downarrow \text { WT marginally associated with maternal reports of } \\
\text { sleep problems }\end{array}$ \\
\hline $\begin{array}{l}\text { St James-Roberts and Peachey } \\
\text { (2011) }\end{array}$ & UK/ Denmark & L & Crying & $\begin{array}{l}610 \\
233 \\
1 \text { and } 3 \text { months }\end{array}$ & Q & NW & $\begin{array}{l}\uparrow N W \text { at } 3 \text { months associated with prolonged crying at } 1 \\
\text { month in one study but not in another }\end{array}$ \\
\hline DeLeon and Karraker (2007) & USA & CS & Crying & $\begin{array}{l}41 \\
9 \text { months }\end{array}$ & $\mathrm{D}$ & $\begin{array}{l}\text { SD ( } 24 \text { h, day, night), } \\
\text { WASO, NW, SP (day) }\end{array}$ & $\begin{array}{l}\downarrow \text { SD (night), } \uparrow \text { WASO, } \uparrow \text { NW at } 9 \text { months associated with } \uparrow \\
\text { daytime crying } \\
\text { SD }(24 \mathrm{~h} \text {, day), and SP (day) at } 9 \text { months not associated with } \\
\text { daytime crying }\end{array}$ \\
\hline Harrison (2004) & UK & L & Crying & $\begin{array}{l}66 \\
1,2,3 \text { months }\end{array}$ & $\mathrm{D}$ & SD (24 h, night) & $\begin{array}{l}\text { SD ( } 24 \mathrm{~h} \text {, night) not associated with crying } \\
\downarrow \text { SD ( } 24 \mathrm{~h} \text {, night) at } 1 \text { month, but not at } 2 \text { and } 3 \text { months } \\
\text { associated with } \uparrow \text { evening crying }\end{array}$ \\
\hline Lee (2000) & Korea & CS & Crying & $\begin{array}{l}188 \\
0-4 \text { months }\end{array}$ & D & SD (24 h) & $\downarrow \mathrm{SD}(24 \mathrm{~h})$ at $0-4$ months associated with $\uparrow$ cry duration \\
\hline
\end{tabular}



Hurry (1997)

UK

Non-associated factors

Agarwal, Gupta, Pushkarna, and India

Bhargava (2000)

Johnson, McMahon, and Gibson Australia

(2014)

Butler, Moore, and Mindell (2016) USA
St James-Roberts, Conroy, and

CS Crying

CS Massage and use of oil

L Conception mode

CS Sleep aid

month

134

7 months

0-11 months

L Sleep aid

30

$0-1,2-3,5-6$

and Horne (2014)

Burnham, Goodlin-Jones, Gaylor, USA and Anders (2002)

Ball (2007)

UK

80

months
L Sleep aid

CS Sleep arrangements (twins)
D

D

D

Q
SD (24 h, day, night), WASO

$\downarrow \mathrm{SD}$ (24 h, day) at 1 months in persistent than in moderate criers

SD (night) and WASO at 1 month not associated with crying

SD (24 h, day, night), SD ( $24 \mathrm{~h}$, day, night), NW and SP (24-h, day) at 1 month not NW, SP (24 h, day) associated with massage and use of oil

SD $(24 \mathrm{~h}) \quad S D(24 \mathrm{~h})$ at 7 months not associated with conception mode

SD (day, night), NW, $\uparrow$ NW at 0-11 months associated with pacifier use and $\uparrow$ LSP

SP (day), LSP

(night), BT, WT

(night) at 0-11 months associated with finger suck

$\uparrow \mathrm{SD}$ (day) at 0-11 months associated with non-suck and pacifier use

SD (night) and SP (day), BT and WT at 0-11 months not associated with sleep aid

$P \quad$ SD (day), SP (day)

SD (day) and SP (day) at 0-1, 2-3, and 5-6 months not associated with sleep aid

V

$1,3,6,9$, and 12

$1-3$ months

V SD (night), NW
SD (night), NW, and LSP (night) not associated with sleep aid

SD (night), NW, LSP

(night)
SD (night) and NW at 1-3 months not associated with sleep arrangements in twin infants

Design: CS Cross-sectional; L Longitudinal.

Sleep measures: A Actigraphy; D Diary; EEG Electro encephalography I Interview; MMS Motility Monitor System; NS Not Specified; O Observation; P Polysmonogrpahy; Q Questionnaire; V Video recording. Sleep-wake behaviour: BT Bedtime; LSP Longest sleep period; SL Sleep latency; NW Night wakings; SD Sleep duration; SE Sleep efficiency; SP Sleep periods; WT Wake Time; WASO Wake After Sleep Onset. 
Table 2. Factors positively and negatively affected by sleep-wake behaviour during the first 12 months of life.

\begin{tabular}{|c|c|c|c|c|c|c|c|}
\hline First author Year of publication & Country & Design & Associated factor & $\begin{array}{c}N \\
\text { Age } \\
\text { (months) }\end{array}$ & Sleep measure & $\begin{array}{l}\text { Sleep-wake } \\
\text { behaviour }\end{array}$ & Main result \\
\hline \multicolumn{8}{|l|}{ Positive factors } \\
\hline McDaniel and Teti (2012) & USA & L & Co-parenting & $\begin{array}{l}150 \\
1 \text { and } 3 \\
\text { months }\end{array}$ & $\mathrm{D}$ & NW & $\begin{array}{l}\uparrow N W \text { at } 1 \text { and } 3 \text { months indirectly predicted } \downarrow \text { co-parenting } \\
\text { quality }\end{array}$ \\
\hline \multicolumn{8}{|l|}{ Negative factors } \\
\hline Tikotzky et al. (2015) & Israel & $\mathrm{L}$ & $\begin{array}{l}\text { Parental bedtime } \\
\text { involvement }\end{array}$ & $\begin{array}{l}57 \\
3 \text { and } 6 \\
\text { months }\end{array}$ & $\begin{array}{l}\text { Combined } \\
\text { measures: A, D, } \\
\text { and Q }\end{array}$ & SD (night), NW, SE & $\begin{array}{l}\text { SD (night), and SE at } 3 \text { and } 6 \text { months and NW at } 3 \text { months } \\
\text { not associated with nighttime paternal involvement at } 6 \\
\text { months }\end{array}$ \\
\hline Johnson et al. (2014) & Australia & $\mathrm{L}$ & $\begin{array}{l}\text { Parental bedtime } \\
\text { involvement }\end{array}$ & $\begin{array}{l}134 \\
7 \text { months }\end{array}$ & D & SD (24 h) & $\begin{array}{l}\downarrow \text { SD }(24 \mathrm{~h}) \text { at } 7 \text { months associated with } \uparrow \text { maternal bedtime } \\
\text { involvement at } 18 \text { months }\end{array}$ \\
\hline Tikotzky and Shaashua (2012) & Israel & L & $\begin{array}{l}\text { Parental bedtime } \\
\text { involvement }\end{array}$ & $\begin{array}{l}71 \\
12 \text { months }\end{array}$ & A & NW & $\begin{array}{l}\uparrow \mathrm{NW} \text { at } 12 \text { months associated with } \uparrow \text { parental bedtime } \\
\text { involvement at } 4 \text { years }\end{array}$ \\
\hline Sharkey et al. (2016) & USA & L & $\begin{array}{l}\text { Parental mental } \\
\text { health }\end{array}$ & $\begin{array}{l}31 \\
0,1 \text {, and } 4 \\
\text { months }\end{array}$ & $\mathrm{D}$ & $\begin{array}{l}\text { SD }(24 \mathrm{~h}), \mathrm{SP} \\
\quad(24 \mathrm{~h})\end{array}$ & $\begin{array}{l}\uparrow \mathrm{SP}(24 \mathrm{~h}) \text { at } 0 \text { and } 1 \text { month associated with } \uparrow \text { maternal } \\
\text { depressive symptoms } \\
\downarrow \text { SD }(24 \mathrm{~h}) \text { at } 0 \text { and } 1 \text { month associated with } \uparrow \text { maternal } \\
\text { stressors at } 4 \text { months }\end{array}$ \\
\hline McDaniel and Teti (2012) & USA & L & $\begin{array}{l}\text { Parental mental } \\
\text { health }\end{array}$ & $\begin{array}{l}150 \\
1 \text { and } 3 \\
\text { months }\end{array}$ & $\mathrm{D}$ & NW & $\begin{array}{l}\uparrow \mathrm{NW} \text { at } 1 \text { and } 3 \text { months indirectly predicted } \uparrow \text { maternal } \\
\text { depression }\end{array}$ \\
\hline Klingenberg et al. (2013) & Denmark & L & Physical growth & $\begin{array}{l}311 \\
9 \text { months }\end{array}$ & Q & SD (24 h, night) & $\begin{array}{l}\text { SD ( } 24 \mathrm{~h} \text {, night) at } 9 \text { months not associated with adiposity } \\
\text { indicators at } 36 \text { months }\end{array}$ \\
\hline Gillman et al. (2008) & USA & L & Physical growth & $\begin{array}{l}1100 \\
6 \text { and } 12 \\
\text { months }\end{array}$ & Q & SD $(24 \mathrm{~h})$ & $\begin{array}{l}\downarrow \text { SD }(24 \mathrm{~h}) \text { at } 6 \text { and } 12 \text { months associated with } \uparrow \text { risk of } \\
\text { overweight at } 3 \text { years }\end{array}$ \\
\hline Dionne et al. (2011) & Canada & L & $\begin{array}{l}\text { Mental } \\
\text { development }\end{array}$ & $\begin{array}{l}1029 \\
6 \text { months }\end{array}$ & 1 & LSP (day, night) & $\begin{array}{l}\uparrow \text { LSP (day) at } 6 \text { months associated with } \downarrow \text { language outcome } \\
\text { at } 18,30 \text { and } 60 \text { months } \\
\text { LSP (night) at } 6 \text { months not associated with language } \\
\text { outcome at } 18,30 \text { and } 60 \text { months }\end{array}$ \\
\hline $\begin{array}{l}\text { Bernier, Carlson, Bordeleau, } \\
\text { and Carrier (2010) }\end{array}$ & Canada & L & $\begin{array}{l}\text { Mental } \\
\text { development }\end{array}$ & $\begin{array}{l}60 \\
12 \text { months }\end{array}$ & $\mathrm{D}$ & SD (24 h), NW, SE & $\begin{array}{l}\uparrow \text { SE at } 12 \text { months associated with } \uparrow \text { performance on } \\
\text { executive tasks at } 26 \text { months (but not at } 18 \text { months) } \\
\text { SD ( } 24 \mathrm{~h} \text { ) and NW at } 12 \text { months not associated performance } \\
\text { on executive tasks at } 18 \text { and } 26 \text { months }\end{array}$ \\
\hline Bouvette-Turcot et al. (2015) & Canada & L & $\begin{array}{l}\text { Social emotional } \\
\text { problems }\end{array}$ & $\begin{array}{l}209 \\
6 \text { and } 12 \\
\text { months }\end{array}$ & Q & SD (24 h) & $\begin{array}{l}\downarrow \text { SD }(24 \mathrm{~h}) \text { from } 6 \text { to } 36 \text { months associated with negative } \\
\text { emotionality/behavioural dysregulation at } 36 \text { months in } \\
\text { infants with } 5 \text {-HTTLPR allele }\end{array}$ \\
\hline Sadeh et al. (2015) & Israel & L & $\begin{array}{l}\text { Social emotional } \\
\text { problems }\end{array}$ & $\begin{array}{l}43 \\
12 \text { months }\end{array}$ & A & SD (night), NW, SE & $\begin{array}{l}\uparrow \mathrm{NW} \text { and } \downarrow \text { SE at } 12 \text { months associated with } \uparrow \text { attention } \\
\text { regulation and behaviour problems at } 3-4 \text { years } \\
\text { SD (night) at } 12 \text { months not associated with attention } \\
\text { regulation and behaviour problems at 3-4 years }\end{array}$ \\
\hline
\end{tabular}




\begin{tabular}{|c|c|c|c|c|c|c|c|}
\hline First author Year of publication & Country & Design & Associated factor & $\begin{array}{c}N \\
\text { Age } \\
\text { (months) }\end{array}$ & Sleep measure & $\begin{array}{l}\text { Sleep-wake } \\
\text { behaviour }\end{array}$ & Main result \\
\hline Saenz et al. (2015) & USA & $\mathrm{L}$ & $\begin{array}{l}\text { Social emotional } \\
\text { problems }\end{array}$ & $\begin{array}{l}47 \\
3-4 \text { months }\end{array}$ & $A$ & SD (night), SE & $\begin{array}{l}\downarrow \text { SD (night) at 3-4 months associated with } \uparrow \text { externalizing } \\
\text { problems and autism spectrum disorder behaviors at 18- } \\
24 \text { months in girls } \\
\text { SD (night) at 3-4 months not associated with internalizing } \\
\text { and dysregulation problems } \\
\text { SE 3-4 months not associated with social emotional } \\
\text { problems }\end{array}$ \\
\hline Scott et al. (2013) & UK & L & $\begin{array}{l}\text { Social emotional } \\
\text { problems }\end{array}$ & $\begin{array}{l}8195 \\
6 \text { months }\end{array}$ & Q & SD (night), NW & $\begin{array}{l}\downarrow \text { SD (night) at } 6 \text { months associated with attention deficit } \\
\text { hyperactivity disorder } \\
\text { NW at } 6 \text { months not associated with attention deficit } \\
\text { hyperactivity disorder }\end{array}$ \\
\hline Bordeleau et al. (2012) & Canada & $\mathrm{L}$ & $\begin{array}{l}\text { Social emotional } \\
\text { problems }\end{array}$ & $\begin{array}{l}55 \\
12 \text { months }\end{array}$ & $\mathrm{D}$ & SD (24 h, night) & $\begin{array}{l}\text { SD ( } 24 \mathrm{~h} \text {, night) at } 12 \text { months not associated with } \\
\text { externalizing and internalizing symptoms at } 4 \text { years }\end{array}$ \\
\hline Jansen et al. (2011) & The Netherlands & L & $\begin{array}{l}\text { Social emotional } \\
\text { problems }\end{array}$ & $\begin{array}{l}4782 \\
2 \text { months }\end{array}$ & Q & SD (24 h), NW & $\begin{array}{l}\uparrow N W \text { at } 2 \text { months associated with } \uparrow \text { anxiety and depression } \\
\text { at } 3 \text { years } \\
\text { SD }(24 \text { h) at } 2 \text { months not associated with anxiety and } \\
\text { depression at } 3 \text { years }\end{array}$ \\
\hline $\begin{array}{l}\text { Novosad, Freudigman, and } \\
\text { Thoman (1999) }\end{array}$ & USA & CS & Temperament & $\begin{array}{l}41 \\
0 \text { and } 8 \\
\text { months }\end{array}$ & MMS & $\operatorname{LSP}(24 \mathrm{~h})$ & $\begin{array}{l}\uparrow \mathrm{LSP}(24 \mathrm{~h}) \text { at } 0 \text { months associated with } \downarrow \text { adaptable } \\
\text { temperament at } 8 \text { months }\end{array}$ \\
\hline Hysing et al. (2014) & Norway & $\mathrm{L}$ & Sleep problems & $\begin{array}{l}55831 \\
6 \text { months }\end{array}$ & Q & SD (night), NW & $\begin{array}{l}\downarrow S D \text { (night) at } 6 \text { months associated with } \downarrow \text { SD (night) at } 18 \\
\text { months } \\
\uparrow \text { NW at } 6 \text { months associated with } \uparrow \text { NW at } 18 \text { months }\end{array}$ \\
\hline $\begin{array}{l}\text { Byars, Yolton, Rausch, } \\
\text { Lanphear, and Beebe (2012) }\end{array}$ & USA & $\mathrm{L}$ & Sleep problems & $\begin{array}{l}359 \\
6,12 \text { months }\end{array}$ & Q & $\begin{array}{l}\text { SD (24 h, day), SL } \\
\text { (night), SP (day) }\end{array}$ & $\begin{array}{l}\downarrow \mathrm{SD}(24 \mathrm{~h}), \uparrow \mathrm{SL} \text { (night) at } 6 \text { and } 12 \text { months associated with } \\
\uparrow \text { sleep problems } \\
\text { SD (day) at } 6 \text { and } 12 \text { months not associated with sleep } \\
\text { problems } \\
\downarrow \text { SD }(24 \mathrm{~h}), \uparrow \mathrm{SL} \text { (night) at } 6 \text { and } 12 \text { months associated with } \\
\downarrow \mathrm{SD}(24 \mathrm{~h}), \uparrow \mathrm{SL} \text { (night) at } 24 \text { and } 36 \text { months } \\
\uparrow \mathrm{SP} \text { (day) at } 6 \text { months associated with } \uparrow \mathrm{SP} \text { (day) at } 12 \\
\text { months, but not at } 24 \text { and } 36 \text { months }\end{array}$ \\
\hline Tikotzky and Shaashua (2012) & Israel & L & Sleep problems & $\begin{array}{l}71 \\
12 \text { months }\end{array}$ & $\begin{array}{l}\text { Combined } \\
\text { measures: A, D, } \\
\text { and Q }\end{array}$ & NW, SE & $\begin{array}{l}\uparrow \mathrm{NW} \text { at } 12 \text { months associated with } \uparrow \mathrm{NW}, \downarrow \mathrm{SE} \text {, and } \downarrow \mathrm{SD} \\
\text { (night) at } 4 \text { years } \\
\uparrow \mathrm{SE} \text { at } 12 \text { months associated with } \uparrow \mathrm{NW} \text { (measured by Q) at } \\
4 \text { years } \\
\text { SE at } 12 \text { months not associated with NW (measured by A), SE, } \\
\text { and SD (night) at } 4 \text { years }\end{array}$ \\
\hline
\end{tabular}


Table 2. Continued.

\begin{tabular}{|c|c|c|c|c|c|c|c|}
\hline First author Year of publication & Country & Design & Associated factor & $\begin{array}{c}N \\
\text { Age } \\
\text { (months) }\end{array}$ & Sleep measure & $\begin{array}{l}\text { Sleep-wake } \\
\text { behaviour }\end{array}$ & Main result \\
\hline Palmstierna et al. (2008) & Sweden & $\mathrm{L}$ & Sleep problems & $\begin{array}{l}16467 \\
12 \text { months }\end{array}$ & $Q$ & NW & $\begin{array}{l}\uparrow N W \text { at } 12 \text { months associated with } \uparrow N W \text { at } 3 \text { and } 5 \text { years } \\
\text { and with } \downarrow \text { perceived sleep quality at } 12 \text { months, } 3 \text { and } 5 \\
\text { years }\end{array}$ \\
\hline $\begin{array}{l}\text { Schwichtenberg, Shah, and } \\
\text { Poehlmann (2013) }\end{array}$ & USA & $\mathrm{L}$ & Attachment & $\begin{array}{l}171 \\
4,9 \text { months }\end{array}$ & D & $\begin{array}{l}\text { SD (day, night), } \\
\text { NW, SP (day) }\end{array}$ & $\begin{array}{l}\uparrow \text { SD (day) from } 4 \text { to } 9 \text { months predicted secure attachment } \\
\text { at } 16 \text { months } \\
\text { SD (night) and NW not associated with attachment }\end{array}$ \\
\hline $\begin{array}{l}\text { Beijers, Jansen, Riksen- } \\
\text { Walraven, and de Weerth } \\
\text { (2011) }\end{array}$ & The Netherlands & $\mathrm{L}$ & Attachment & $\begin{array}{l}193 \\
0-6 \text { and } 12 \\
\text { months }\end{array}$ & D & NW & $\begin{array}{l}\uparrow \text { NW at } 0-6 \text { months associated with insecure-resistant } \\
\text { attachment at } 12 \text { months } \\
\downarrow \text { NW at } 6 \text { months associated with avoidant attachment at } 12 \\
\text { months }\end{array}$ \\
\hline
\end{tabular}

Design: CS Cross-sectional; L Longitudinal.

Sleep measures: A Actigraphy; D Diary; EEG Electro encephalography I Interview; MMS Motility Monitor System; NS Not Specified; O Observation; P Polysmonogrpahy; Q Questionnaire; V Video recording.

Sleep-wake behaviour: BT Bedtime; LSP Longest sleep period; SL Sleep latency; NW Night wakings; SD Sleep duration; SE Sleep efficiency; SP Sleep periods; WT Wake Time; WASO Wake After Sleep Onset. 
sleep period (time of the longest sleep episode), bedtime/sleep onset time (hour of sleep start during the night), wake time (waking time in the morning), and sleep efficiency (percentage of sleep during the night from bedtime to wake time).

\section{Results}

\section{Databases search}

After the elimination of duplicates, 8610 relevant papers were identified in the literature search. The examination of titles and abstracts lead to the exclusion of 8244 non-relevant papers and the remaining 366 papers were examined for inclusion/exclusion criteria. Two hundred and twenty papers met one or more exclusion criteria and were excluded. This systematic review included 146 papers. Figure 1 presents a flow diagram of the search.

\section{Articles reviewed}

Studies performed in 25 countries were included. Most of the studies were performed in the United States $(n=42)$, Israel $(n=22)$, UK $(n=15)$, Australia $(n=11)$, or Canada $(n=10)$ and about half of the studies presented a longitudinal design $(n=76)$. Infant sleep-wake behaviour's measures included questionnaire $(n=53)$, diary $(n=33)$, actigraphy $(n=17)$, interview $(n=10)$, video recording $(n=7)$, polysomnography $(n=5)$, observation by experimented researchers $(n=2)$, electroencephalography $(n=1)$, motility monitoring system $(n=1)$, combined measures $(n=15 ; 8$ studies used actigraphy and sleep diary; 4 studies used actigraphy and questionnaire; 2 studies used actigraphy, sleep diary and questionnaire; and 1 study used sleep diary and polysomnography), and 2 studies did not specify sleep-wake behaviour measures. About half of the studies were scored as good $(n=76)$, and the other half as moderate quality $(n=70)$ in the Quality Index.

\section{Factors positively and negatively associated with sleep-wake behaviour}

The following factors emerged as positively associated with sleep-wake behaviour during the first 12 months of life: being a first-time infant in $4 / 6$ studies, Caucasian ethnicity in $3 / 3$ studies, higher parental educational/health literacy level in $3 / 5$ studies, lower sensory and environmental stimuli in $1 / 1$ study, better genetic/environmental influences in $1 / 1$ study, outdoor environment in $1 / 1$ study,

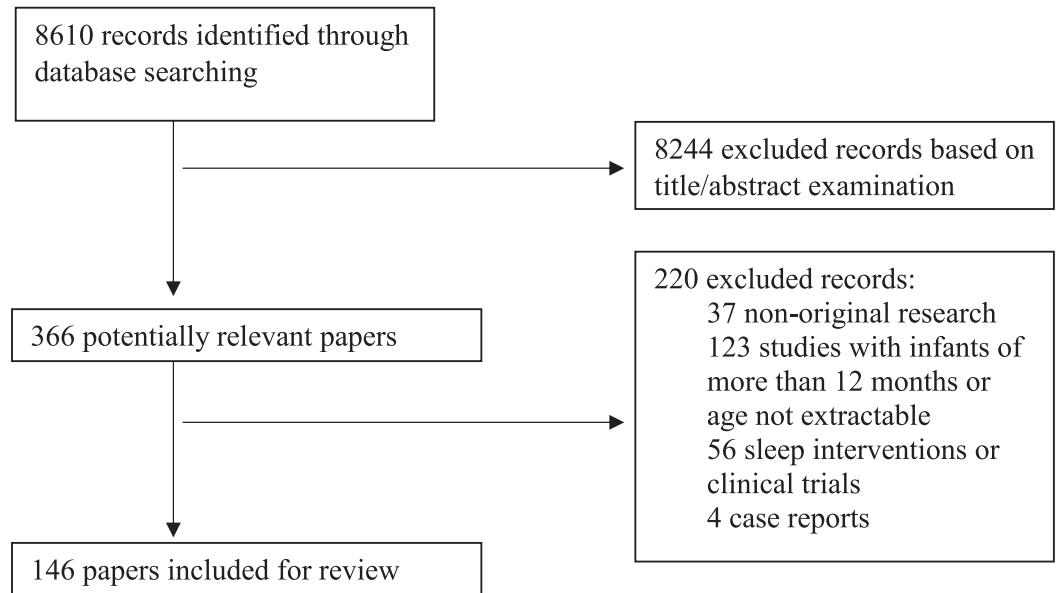

Figure 1 . Search strategy flow chart. 
sleeping in prone position in 1/1 study, wearing Ö4 fabric in 1/1 study, regulated cortisol and melatonin levels in $4 / 4$ studies, infant secure attachment patterns in 2/3 studies, and better mother-infant relationship in $3 / 3$ studies (see Table 1 ).

On the other hand, the following factors emerged as negatively associated with sleep-wake behaviour during the first 12 months of life: male gender in 10/12 studies, older maternal age in $2 / 2$ studies, single/divorced parents in $2 / 2$ studies, non-native parents in $1 / 1$ study, lower socio-economic status in $3 / 5$ studies, winter months in $1 / 1$ study, urban environments in $1 / 1$ study, less exposure to light in $1 / 1$ study, and more exposure to maternal smoking in 1/1 study, gestational exposure to smoke, alcohol, substances, namely alcohol, tobacco, cocaine, marijuana, and methamphetamine, and stressful events in 3/4 studies, planned C-section in 1/1 study, and preterm delivery in 4/4 studies, breastfeeding in 22/27 studies, earlier introduction of solids, higher number of feedings, and diet components in 3/4 studies, later bedtimes, less bedtime activities, and television and media exposure in 5/5 studies, more parental bedtime involvement (characterized by parental presence at bedtime, nursing or rocking at bedtime, putting the infant in crib asleep, or feeding back to sleep) in $8 / 8$ studies, bed-sharing or co-sleeping in 10/11 studies, cloth diapers in 1/1 study, maternal depression, stress, or fatigue and family dysfunction and stress in 17/18 studies, worse parental sleep in $7 / 7$ studies, lower parental involvement in $4 / 5$ studies, emergence of crawling in $4 / 6$ studies, weight gain in $3 / 4$ studies, colic in $2 / 2$ studies, health problems in $5 / 6$ studies, lower scores in mental development tasks in $6 / 7$ studies, social and emotional problems in $6 / 7$ studies, difficult temperament in 10/10 studies, sleep problems in 3/3 studies, and crying in 5/5 studies (see Table 1).

Massage and use of oil, conception mode, sleep aid, and sleep arrangements in twins were not associated with infant sleep-wake behaviour (see Table 1).

\section{Factors positively and negatively affected by sleep-wake behaviour}

Sleep-wake behaviour during the first 12 months of life was shown to positively affect co-parenting quality in $1 / 1$ study and negatively affect parental bedtime involvement in $2 / 3$ studies, maternal mental health in $2 / 2$ studies, obesity in $1 / 2$ study, performance in mental tasks in $2 / 2$ studies, social emotional problems in 5/6 studies, temperament in $1 / 1$ study, sleep problems in $4 / 4$ studies, and attachment in 2/2 studies (see Table 2).

\section{Discussion}

This study aimed to provide a systematic review of the literature on associated factors with sleepwake behaviour during the first 12 months of life. This systematic review considered different sleep-wake behaviours and included studies performed over the last 20 years in 25 different countries.

\section{Factors positively and negatively associated with sleep-wake behaviour}

The most consistent factors found in literature as being positively associated with the different sleepwake behaviours during the first 12 months of life were: being a first-time infant, Caucasian ethnicity, the regulation of the HPA axis, secure attachment pattern and a positive mother-infant relationship. The other factors were either poorly explored or provided inconsistent results across the different studies.

Being a first-time infant was associated with longer sleep duration and less night wakings (Kozyrskyj et al., 2009; Santos et al., 2008; So et al., 2007). Results from studies with the smallest samples either suggested no differences in the different sleep-wake behaviours that were analyzed (Kaley et al., 2012; Quillin, 1997; So et al., 2007) or a tendency for longer sleep duration in non-first-time infants (Kaley et al., 2012). When the number of siblings was assessed, different results were found, namely more night wakings in infants with more siblings in one study (Kozyrskyj et al., 
2009) and no differences in another study (Blair et al., 2012). Studies addressing birth order also differed regarding the time of sleep-wake behaviour assessment. While during the first months, results were not consistent (Kaley et al., 2012; Quillin, 1997; So et al., 2007), in the second half of the first year, non-first-time infants were shown to present less sleep duration during the night (So et al., 2007), and more night wakings (Kozyrskyj et al., 2009; Santos et al., 2008). As the most important changes in sleep-wake behaviour occur during the first 3 months (Burnham et al., 2002; Henderson, France, \& Blampied, 2011), differences in birth order may not be easily seen in the first months.

Caucasian ethnicity was consistently associated with longer sleep duration during the 24-hour period and more night wakings when compared with non-caucasian ethnicity (Blair et al., 2012; Nevarez et al., 2010; Santos et al., 2012). This result is consistent with previous research showing cultural differences in sleep-wake behaviours between Caucasian and Asian infants (Mindell, Sadeh, Wiegand, How, \& Goh, 2010).

Although some inconsistent results, a secure attachment was associated with more regulated sleep-wake behaviour, characterized by more sleep duration during the night, less night wakings, and a longer longest sleep period in 2 studies (Pennestri et al., 2015; Zentall et al., 2012). This may be explained by the fact that secure infants are more able to deal with the mother separation during the night and rely on mother's availability, presenting a more regulated sleep-wake behaviour during the night (Zentall et al., 2012). Regarding mother-infant interaction, different results have been found. A positive mother-infant relationship was associated with a higher increase in the longest sleep periods (de Graag et al., 2012) and with more night wakings, but not with sleep duration during the night and bedtime (Scher, 2001b). Researchers studying preterm infants showed the importance of sleep-wake behaviors during the day in association with the mother-infant relationship (Schwichtenberg et al., 2011).

Although few studies addressed infant hormonal factors, results suggested a trend for an association between a regulation of the HPA axis and less night wakings (Lucas-Thompson et al., 2009) and more sleep during the night (Lucas-Thompson et al., 2009). Results from a recent study were controversial, however, this study was conducted with infants with colic (Brand et al., 2011), and colic were shown to be associated with infant sleep-wake behaviour in previous research (Kirjavainen et al., 2001; White et al., 2000). Furthermore, lower morning melatonin levels were also associated with increased sleep duration during the night in one study (Shinohara \& Kodama, 2011).

The most consistent factors negatively associated with different sleep-wake behaviours during the first 12 months of life were: male gender, breastfeeding, bedtime routines, bed-sharing, parental mental health, parental sleep, and infant social-emotional and sleep problems. Other factors were also negatively associated with sleep-wake behaviour, namely lower socio-economic status, preterm birth, crawling onset, difficult temperament, and crying. However, considering the paucity of studies or the inconsistent results, more research is needed.

Male gender was consistently associated with shorter sleep duration, more night wakings, less sleep efficiency, and shorter longest sleep periods during the first 12 months of life. Interestingly, results from studies performed in Australia suggested no gender differences in the different sleepwake behaviours that were analyzed (Kozyrskyj et al., 2009; So et al., 2007), which can underlie some cultural characteristics. Non-Australian studies, whose results showed no gender differences in the different infant sleep-wake behaviours, were conducted with small samples and most of them assessed age ranges rather than specific ages (Goodlin-Jones et al., 2001; Kaley et al., 2012; Lampl \& Johnson, 2011; Saenz et al., 2015; Thomas \& Foreman, 2005). Considering that infant sleep-wake behaviour during the first months is marked by both individual and developmental changes (Figueiredo et al., 2016), the use of small sample sizes or age ranges could difficult the assessment of gender differences (Weinraub et al., 2012).

Breastfeeding was consistently associated with more night wakings (Anuntaseree et al., 2008; Ball, 2003; DeLeon \& Karraker, 2007; Engler et al., 2012; Galbally et al., 2013; Huang et al., 2016; Hughes et al., 2015; Hysing et al., 2014; Kaley et al., 2012; Kozyrskyj et al., 2009; Mindell et al., 2009, 2012; Ramamurthy et al., 2012; Schwichtenberg \& Poehlmann, 2009; Tikotzky et al., 2015; Wolke et al., 1998). This 
is congruent with previous literature showing that the quick digestion of breast milk could lead to a more fragmented sleep in breastfed infants (Burness, 1979). However, it is not clear the association between feeding method and other sleep-wake behaviours, namely sleep duration, sleep periods or longest sleep period (Cubero et al., 2005; DeLeon \& Karraker, 2007; Demirci et al., 2012; Engler et al., 2012; Huang et al., 2016; Hughes et al., 2015; Kaley et al., 2012; Lampl \& Johnson, 2011; Lee, 2000; Mindell et al., 2012; Morgan et al., 2004; Nevarez et al., 2010; Quillin \& Glenn, 2004; Ramamurthy et al., 2012; Schwichtenberg \& Poehlmann, 2009; Thomas, 2000).

Less arousing activities, no reading, late betimes, no bedtime routine, and television and media exposure before bedtime were associated with shorter sleep duration during the night and more night wakings (Cespedes et al., 2014; Mindell et al., 2009; Nevarez et al., 2010; Philbrook \& Teti, 2016; Vijakkhana et al., 2015). This is congruent with findings that show that sleep hygiene is associated with infant sleep-wake behaviour (Mindell et al., 2009). Furthermore, higher parental bedtime involvement, characterized by parental presence at bedtime, nursing or rocking at bedtime, putting the infant in crib asleep, or feeding back to sleep were also associated with shorter sleep duration and longest sleep periods, more sleep latency during the night and more night wakings (Anuntaseree et al., 2008; DeLeon \& Karraker, 2007; Goodlin-Jones et al., 2001; Huang et al., 2016; Mindell et al., 2009; Philbrook \& Teti, 2016; Ramamurthy et al., 2012; Tikotzky \& Sadeh, 2009; Touchette et al., 2005). These findings may be explained within the context of the transactional model that argues that parental behaviours are the most immediate and direct factor influencing infant sleep-wake behaviours (Sadeh et al., 2010). The level of parental involvement at bedtime is crucial to the development of the infant's abilities of self-soothing and self-regulation (Sadeh et al., 2010). In the presence of a low parental involvement at bedtime, the infant is taught to self-sooth and self-regulate its sleep-wake behaviors, while in the presence of high parental involvement at bedtime, the infant is not able to develop these skills (Sadeh et al., 2010).

Sleep arrangements (characterized by bed-sharing or co-sleeping) were consistently associated with shorter sleep duration during the night and more night wakings (Bruni et al., 2014; DeLeon \& Karraker, 2007; Guyer et al., 2015; Jenni et al., 2005; Mao et al., 2004; Möllborg et al., 2011; Philbrook \& Teti, 2016; Santos et al., 2008; Touchette et al., 2005). Bed-sharing/co-sleeping promotes higher physical contact with the parents and that could interfere with the infant's ability of self-comforting, causing more difficulties in the ability of the infant to return to sleep after night wakings (Green, Groves, \& Tegano, 2004; Morrell \& Steele, 2003). Moreover, previous research also showed an association between co-sleeping/bed-sharing and infant's dysregulation, breastfeeding and putting the infant in bed asleep, arguing that co-sleeping/bed-sharing could be used as a parent's strategy to deal with the infant's characteristics and the feeding method (DeLeon \& Karraker, 2007). Only 1 study showed no associations between sleep arrangements and infant sleep-wake behaviour (Baddock et al., 2006). However, this study analyzed differences between bed-sharing and cot-sleeping, being cot-sleeping defined as sleeping in a cot, that is usually adjacent to the parent's bed (Baddock et al., 2006). Furthermore, this study was performed in New Zealand, where the lowest bed-sharing rates were shown (Mindell et al., 2009). No association between sleep arrangements and sleep-wake behaviour, when measured by actigraphy, was found in another study (Volkovich et al., 2015). However, the analyses of this study were controlled for feeding method, which has been shown to be associated with sleep arrangements in previous research (DeLeon \& Karraker, 2007). Interestingly, although a recent study, performed in China, found more sleep duration during the night in independent sleeping infants, no association was shown between sleep arrangements and night wakings (Huang et al., 2016).

Parental mental health, especially maternal depression and fatigue and family dysfunction and stress have been associated with shorter sleep duration (day, night and 24-hour period) and longest sleep period (night), and more night wakings and WASO (Armitage et al., 2009; Baird et al., 2009; Brand et al., 2014; Dennis \& Ross, 2005; Goldberg et al., 2013; Gress-Smith et al., 2012; Hughes et al., 2015; Karraker \& Young, 2007; Loutzenhiser et al., 2015; Mao et al., 2011; Nevarez et al., 2010; O'Connor et al., 2007; Piteo et al., 2013; Saenz et al., 2015; Sinai \& Tikotzky, 2012; 
Sorondo \& Reeb-Sutherland, 2015; Warren et al., 2006). Depressed mothers may be less able to deal with family actives, namely infant sleep-wake behaviour (Piteo et al., 2013). However, the results of 6 studies indicated no association between parental mental health and the different infant sleep-wake behaviours that were analyzed (Armitage et al., 2009; Dennis \& Ross, 2005; O'Connor et al., 2007; Sinai \& Tikotzky, 2012; Sorondo \& Reeb-Sutherland, 2015; Thomas \& Spieker, 2016). Five of these studies used measures based on parental reports (Dennis \& Ross, 2005; O'Connor et al., 2007; Sinai \& Tikotzky, 2012; Sorondo \& Reeb-Sutherland, 2015; Thomas \& Spieker, 2016). Curiously, maternal mental health was not associated with infant sleep-wake behaviour during the day in the only study that used actigraphy (Armitage et al., 2009).

Parental sleep was also associated with infant sleep-wake behaviour, namely sleep duration, sleep periods, longest sleep period, night wakings, sleep efficiency and bedtimes (Brand et al., 2014; Demirci et al., 2012; Sharkey et al., 2016; Sinai \& Tikotzky, 2012; Thomas \& Foreman, 2005; Thomas \& Spieker, 2016; Tikotzky et al., 2015). Parents, especially mothers, presenting poor sleep could be either more stressed during the day or more actively involved in infants' soothing after a night waking which may interfere with infant's ability of self-soothing (Tikotzky \& Sadeh, 2010; Tikotzky et al., 2015). Parental sleep was not associated with the different infant sleep-wake behaviours that were analyzed in 3 other studies (Sharkey et al., 2016; Sinai \& Tikotzky, 2012; Thomas \& Foreman, 2005). These studies were performed with small samples and 1 of them comprised a sample with a history of major depressive disorder (Sharkey et al., 2016). Furthermore, these authors found no association between parental sleep and infant sleep-wake behaviour during the day and the 24-hour period, which may suggest that parental sleep is more associated with infant sleep-wake behaviour during the night.

The presence of social and emotional problems (DeLeon \& Karraker, 2007; Landau et al., 2010; McGeorge et al., 2015; Scher, 2008; Scher \& Blumberg, 1999; Spruyt et al., 2008), with exception of autism (Humphreys et al., 2014 ), was also associated with worse sleep-wake behaviour.

More night wakings, sleep periods and sleep latency and shorter sleep duration (night and 24hour period) were associated with sleep problems (Gibson, Gander, et al., 2012; Hiscock \& Wake, 2001; Scher et al., 1998). In fact, literature has been suggesting that some sleep-wake behaviours, especially night wakings and short sleep duration, are already considered as sleep problems during the first 12 months and remain a concern for parents further in infant development (Bayer et al., 2007).

Lower socio-economic status, larger households, and maternal employment were associated with shorter sleep duration and longest sleep period, and more night wakings and sleep periods (Fouts et al., 2007; Nevarez et al., 2010; Santos et al., 2008). Considering the paucity of studies and that different parent's socio-demographic and socio-economic factors were assessed, these variables need a more extensive study to a better understanding of their association with sleep-wake behaviour during the first 12 months.

Although, during the first 6 months, preterm infants sleep more (day, and night), in longer periods (night), and present more night wakings (Guyer et al., 2015; Huang et al., 2014) at 12 months, results are not consistent across the reviewed studies (Asaka \& Takada, 2010; Kozyrskyj et al., 2009). Preterm infants achieve the 24-hour sleep-wake rhythms earlier than term infants (Guyer et al., 2015), and thus the differences between term and preterm infants could be more evident during the first 6 months. However, more research is warranted to better understand the association between gestational age and sleep-wake behaviour during the first 12 months of life.

Although no association was found between motor development and infant-sleep wake behaviour in 2 studies (Gibson, Elder, et al., 2012; Mindell \& Lee, 2015), more night wakings were reported in the emergence of crawling (Atun-Einy \& Scher, 2016; Scher \& Cohen, 2005), especially in infants with early crawling (Scher, 2005a; Scher \& Cohen, 2015). At crawling onset, mothers may be more involved in infant's bedtime or increase their perception of involvement in infant sleep-wake behaviour during the night, which may increase infant's night wakings (Scher \& Cohen, 2005). 
Results also suggest a trend for an association between easy temperament and less night wakings (Bayer et al., 2007; Mindell \& Lee, 2015; Palmstierna et al., 2008; Scher et al., 1998), less WASO (Sorondo \& Reeb-Sutherland, 2015), less sleep latency (Gibson, Gander, et al., 2012; Sorondo \& Reeb-Sutherland, 2015), longer sleep duration (day, night, and 24-hour) (Gibson, Gander, et al., 2012; Kaley et al., 2012; Mindell \& Lee, 2015; Scher et al., 1998; Spruyt et al., 2008; Touchette et al., 2005), less sleep periods (Mindell \& Lee, 2015), more sleep efficiency (Scher, 2001a; Scher et al., 1998), earlier bedtime (Scher et al., 1998) and later wake time (Gibson, Gander, et al., 2012; Mindell \& Lee, 2015). Previous authors hypothesized that these associations may be influenced by parents' perceptions (Mindell \& Lee, 2015; Spruyt et al., 2008). Moreover, temperament dimensions were not associated with the different infant sleep-wake behaviors that were analyzed (Gibson, Gander, et al., 2012; Kaley et al., 2012; Mindell \& Lee, 2015; Scher, 2001a). However, these results seem to suggest that infants with a more difficult temperament and negative mood present worse sleepwake behaviours.

Although there is a tendency for an association between crying and less sleep duration and more fragmented sleep (DeLeon \& Karraker, 2007; Harrison, 2004; Lee, 2000; St James-Roberts et al., 1997; St James-Roberts \& Peachey, 2011), this association is not congruent across the different studies. Studies addressing this association used parent's reports to assess both crying and infant-sleep wake behaviour, which may underlie some biased perceptions. Further studies should address this association using actigraphy.

\section{Factors positively and negatively affected by sleep-wake behaviour}

Effects of sleep-wake behaviour were less studied, but worse sleep-wake behaviour were shown to predict later social-emotional (Bernier et al., 2010; Bordeleau et al., 2012; Bouvette-Turcot et al., 2015; Sadeh et al., 2015; Saenz et al., 2015), and sleep problems (Byars et al., 2012; Hysing et al., 2014; Palmstierna et al., 2008; Tikotzky \& Shaashua, 2012).

More night wakings, shorter sleep duration (night and 24-hour period) and less sleep efficiency early in infancy were shown in infants with social and emotional problems, namely attention and emotional regulation and attention deficit hyperactivity disorder (Sadeh et al., 2015; Scott et al., 2013), externalizing problems (Saenz et al., 2015), autism disorder (Saenz et al., 2015), and anxiety and depression symptoms (Jansen et al., 2011). This may indicate that early sleep-wake behaviour is associated with brain maturation (Saenz et al., 2015). Authors of a recent study stated the importance of differential susceptibility in the association between sleep duration and later emotional development, reporting that this association is only present in infants with 5-HTTLPR allele (Bouvette-Turcot et al., 2015). Sleep-wake behaviour was not associated with externalizing problems in 1 study, whose results suggested, however, that sleep duration during the night moderated the association between maternal sensitivity and internalizing/externalizing symptoms (Bordeleau et al., 2012).

Shorter sleep duration, more night wakings, sleep periods and sleep latency were associated with later sleep problems (Byars et al., 2012; Hysing et al., 2014; Palmstierna et al., 2008; Tikotzky \& Shaashua, 2012). This is consistent with the results of a previous study, pointing for the individual stability in infant sleep-wake behaviour over the first 6 months (Figueiredo et al., 2016).

\section{Conclusions}

This systematic review provided information regarding factors associated with the different sleepwake behaviour during the first 12 months of life. Different factors were identified as being positively and negatively associated with different sleep-wake behaviors, namely infant factors, parental and environmental factors. Factors positively and negatively affected by different sleep-wake behaviours during the first 12 months of life were also presented in this systematic review. Moreover, the bidirectional nature of different factors was shown. For example, infant social-emotional and sleep 
problems were shown to be negatively associated with some infant sleep-wake behaviours and were also found to be negatively affected by other infant sleep-wake behaviours.

Methodological differences between the studies seemed to underlie some different results between them, namely: (1) the use of different sleep-wake measures; (2) the study of infants with different ages or age-ranges; (3) the study of samples with different characteristics; (4) the use of different definitions of the studied variables; and (5) the study of different sleep-wake behaviours.

Infant sleep-wake behaviour presents significant developmental changes and individual differences during the first months (Figueiredo et al., 2016), being imperative the assessment of infants in specific ages and the use of large and representative samples. Infant sleep-wake behaviour presents cultural differences (Mindell et al., 2010) and incongruent results were also identified in studies performed in specific countries (e.g. Baddock et al., 2006; Kozyrskyj et al., 2009; So et al., 2007). Considering the homogeneity of some studies' samples, it could be difficult to generalize some results (Field, 2017).

Despite these differences, this systematic review identified several factors associated with the different sleep-wake behaviours during the first 12 months of life.

\section{Disclosure statement}

No potential conflict of interest was reported by the authors.

\section{Funding}

This work was supported by FEDER Funds through the Programa Operacional Factores de Competitividade - COMPETE and by National Funds through FCT - Fundação para a Ciência e a Tecnologia under the project: PTDC/SAU/SAP/116738/ 2010 and SFRH/BD/113005/2015 PhD grant. This study was conducted at Psychology Research Centre (UID/PSI/01662/ 2013), University of Minho, and supported by the Portuguese Foundation for Science and Technology and the Portuguese Ministry of Education and Science through national funds and co-financed by FEDER through COMPETE2020 under the PT2020 Partnership Agreement (POCI-01-0145-FEDER-007653).

\section{Notes on contributors}

Cláudia Castro Dias is a PhD student at the School of Psychology University of Minho, Braga, Portugal.

Bárbara Figueiredo, PhD, is Professor at the School of Psychology, University of Minho, Braga, Portugal.

\section{ORCID}

Cláudia Castro Dias (D) http://orcid.org/0000-0002-5511-457X

Bárbara Figueiredo (iD http://orcid.org/0000-0002-8209-7445

\section{References}

Agarwal, K. N., Gupta, A., Pushkarna, R., \& Bhargava, S. K. (2000). Effects of massage \& use of oil on growth, blood flow \& sleep pattern in infants. Indian Journal of Medical Research, 112, 212-217.

Anuntaseree, W., Mo-Suwan, L., Vasiknanonte, P., Kuasirikul, S., \& Choprapawan, C. (2008). Night waking in Thai infants at 3 months of age: Association between parental practices and infant sleep. Sleep Medicine, 9, 564-571. doi:10.1016/j. sleep.2007.07.009

Anuntaseree, W., Sangsupawanich, P., Osmond, C., Mo-Suwan, L., Vasiknanonte, P., \& Choprapawon, C. (2012). Sleep quality in infants with atopic dermatitis: A community-based, birth cohort study. Asian Pacific Journal of Allergy and Immunology, 30(1), 26-31.

Armitage, R., Flynn, H., Hoffmann, R., Vazquez, D., Lopez, J., \& Marcus, S. (2009). Early developmental changes in sleep in infants: The impact of maternal depression. Sleep, 32, 693-696. doi:10.1093/sleep/32.5.693

Asaka, Y., \& Takada, S. (2010). Activity-based assessment of the sleep behaviors of VLBW preterm infants and full-term infants at around 12 months of age. Brain and Development, 32, 150-155. 
Atun-Einy, O., \& Scher, A. (2016). Sleep disruption and motor development: Does pulling-to-stand impacts sleep-wake regulation? Infant Behavior and Development, 42, 36-44. doi:10.1016/j.infbeh.2015.11.003

Bach, V., Telliez, F., Leke, A., \& Libert, J. P. (2000). Gender-related sleep differences in neonates in thermoneutral and cool environments. Journal of Sleep Research, 9, 249-254. doi:10.1046/j.1365-2869.2000.00206.x

Baddock, S. A., Galland, B. C., Bolton, D. P., Williams, S. M., \& Taylor, B. J. (2006). Differences in infant and parent behaviors during routine bed sharing compared with cot sleeping in the home setting. Pediatrics, 117, 1599-1607. doi:10.1542/ peds.2005-1636

Baird, J., Hill, C. M., Kendrick, T., Inskip, H. M., \& SWS Study Group. (2009). Infant sleep disturbance is associated with preconceptional psychological distress: Findings from the Southampton Women's Survey. Sleep, 32, 566-568. doi:10. 5665/sleep/32.4.566

Ball, H. L. (2003). Breastfeeding, bed-sharing, and infant sleep. Birth (Berkeley, Calif), 30, 181-188. doi:10.1046/j.1523-536X. 2003.00243.x

Ball, H. L. (2007). Together or apart? A behavioural and physiological investigation of sleeping arrangements for twin babies. Midwifery, 23, 404-412. doi:10.1016/j.midw.2006.07.004

Bathory, E., Tomopoulos, S., Rothman, R., Sanders, L., Perrin, E. M., Mendelsohn, A., .. Yin, H. S. (2016). Infant sleep and parent health literacy. Academic Pediatrics, 16, 550-557. doi:10.1016/j.acap.2016.03.004

Bayer, J. K., Hiscock, H., Hampton, A., \& Wake, M. (2007). Sleep problems in young infants and maternal mental and physical health. Journal of Paediatrics and Child Health, 43, 66-73. doi:10.1111/j.1440-1754.2007.01005.x

Beijers, R., Jansen, J., Riksen-Walraven, M., \& de Weerth, C. (2011). Attachment and infant night waking: A longitudinal study from birth through the first year of life. Journal of Developmental \& Behavioral Pediatrics, 32, 635-643. doi:10. 1097/DBP.0b013e318228888d

Bernier, A., Carlson, S. M., Bordeleau, S., \& Carrier, J. (2010). Relations between physiological and cognitive regulatory systems: Infant sleep regulation and subsequent executive functioning. Child Development, 81, 1739-1752. doi:10. 1111/j.1467-8624.2010.01507.x

Bhat, R. Y., Hannam, S., Pressler, R., Rafferty, G. F., Peacock, J. L., \& Greenough, A. (2006). Effect of prone and supine position on sleep, apneas, and arousal in preterm infants. Pediatrics, 118, 101-107. doi:10.1542/peds.2005-1873

Blair, P. S., Humphreys, J. S., Gringras, P., Taheri, S., Scott, N., Emond, A., ... Fleming, P. J. (2012). Childhood sleep duration and associated demographic characteristics in an English cohort. Sleep, 35, 353-360. doi:10.5665/sleep.1694

Bordeleau, S., Bernier, A., \& Carrier, J. (2012). Maternal sensitivity and children's behavior problems: Examining the moderating role of infant sleep duration. Journal of Clinical Child \& Adolescent Psychology, 41, 471-481. doi:10.1080/ 15374416.2012.686101

Bottino, C. J., Rifas-Shiman, S. L., Kleinman, K. P., Oken, E., Redline, S., Gold, D., ... Taveras, E. M. (2012). The association of urbanicity with infant sleep duration. Health \& Place, 18, 1000-1005. doi:10.1016/j.healthplace.2012.06.007

Bouvette-Turcot, A. A., Pluess, M., Bernier, A., Pennestri, M. H., Levitan, R., Sokolowski, M. B., ... Meaney, M. J. (2015). Effects of genotype and sleep on temperament. Pediatrics, 136, e914-e921. doi:10.1542/peds.2015-0080

Brand, S., Furlano, R., Sidler, M., Schulz, J., \& Holsboer-Trachsler, E. (2011). 'Oh, baby, please don't cry!': In infants suffering from infantile colic hypothalamic-pituitary-adrenocortical axis activity is related to poor sleep and increased crying intensity. Neuropsychobiology, 64, 15-23. doi:10.1159/000322456

Brand, S., Furlano, R., Sidler, M., Schulz, J., \& Holsboer-Trachsler, E. (2014). Associations between infants' crying, sleep and cortisol secretion and mother's sleep and well-being. Neuropsychobiology, 69, 39-51. doi:10.1159/000356968

Brown, A., \& Harries, V. (2015). Infant sleep and night feeding patterns during later infancy: Association with breastfeeding frequency, daytime complementary food intake, and infant weight. Breastfeeding Medicine, 10, 246-252. doi:10.1089/ bfm.2014.0153

Bruni, O., Baumgartner, E., Sette, S., Ancona, M., Caso, G., Di Cosimo, M. E., ... Ferri, R. (2014). Longitudinal study of sleep behavior in normal infants during the first year of life. Journal of Clinical Sleep Medicine, 10, 1119-1127. doi:10.5664/ jcsm. 4114

Burness, N. (1979). Infant feeding. In V. C. Vaughan, R. J. McKay, \& R. E. Behrman (Eds.), Nelson textbook of pediatrics (pp. 199). Philadelphia, PA: WB Saunders Co.

Burnham, M. M., Goodlin-Jones, B. L., Gaylor, E. E., \& Anders, T. F. (2002). Nighttime sleep-wake patterns and self-soothing from birth to one year of age: A longitudinal intervention study. Journal of Child Psychology and Psychiatry, 43, 713725. doi:10.1111/1469-7610.00076

Butler, R., Moore, M., \& Mindell, J. A. (2016). Pacifier use, finger sucking, and infant sleep. Behavioral Sleep Medicine, 14, 615-623. doi:10.1080/15402002.2015.1048451

Byars, K. C., Yolton, K., Rausch, J., Lanphear, B., \& Beebe, D. W. (2012). Prevalence, patterns, and persistence of sleep problems in the first 3 years of life. Pediatrics, 129, e276-e284. doi:10.1542/peds.2011-0372

Cespedes, E. M., Gillman, M. W., Kleinman, K., Rifas-Shiman, S. L., Redline, S., \& Taveras, E. M. (2014). Television viewing, bedroom television, and sleep duration from infancy to mid-childhood. Pediatrics, 133, e1163-e1171. doi:10.1542/ peds.2013-3998

Cohen, D., Atun-Einy, O., \& Scher, A. (2012). Seasonal effect on infants' sleep regulation: A preliminary study in a Mediterranean climate. Chronobiology International, 29, 1352-1357. doi:10.3109/07420528.2012.728654 
Cubero, J., Valero, V., Sánchez, J., Rivero, M., Parvez, H., Rodríguez, A. B., \& Barriga, C. (2005). The circadian rhythm of tryptophan in breast milk affects the rhythms of 6-sulfatoxymelatonin and sleep in newborn. Neuroendocrinology Letters, 26(6), 657-662.

de Graag, J. A., Cox, R. F., Hasselman, F., Jansen, J., \& de Weerth, C. (2012). Functioning within a relationship: Mother-infant synchrony and infant sleep. Infant Behavior and Development, 35, 252-263. doi:10.1016/j.infbeh.2011.12.006

DeLeon, C. W., \& Karraker, K. H. (2007). Intrinsic and extrinsic factors associated with night waking in 9-month-old infants. Infant Behavior and Development, 30, 596-605. doi:10.1016/j.infbeh.2007.03.009

Demirci, J. R., Braxter, B. J., \& Chasens, E. R. (2012). Breastfeeding and short sleep duration in mothers and 6-11-month-old infants. Infant Behavior and Development, 35, 884-886. doi:10.1016/j.infbeh.2012.06.005

Dennis, C. L., \& Ross, L. (2005). Relationships among infant sleep patterns, maternal fatigue, and development of depressive symptomatology. Birth (Berkeley, Calif), 32, 187-193. doi:10.1111/j.0730-7659.2005.00368.x

Dionne, G., Touchette, E., Forget-Dubois, N., Petit, D., Tremblay, R. E., Montplaisir, J. Y., \& Boivin, M. (2011). Associations between sleep-wake consolidation and language development in early childhood: A longitudinal twin study. Sleep, 34, 987-995. doi:10.5665/SLEEP.1148

Downs, S. H., \& Black, N. (1998). The feasibility of creating a checklist for the assessment of the methodological quality both of randomised and non-randomised studies of health care interventions. Journal of Epidemiology \& Community Health, 52, 377-384. doi:10.1136/jech.52.6.377

Engler, A. C., Hadash, A., Shehadeh, N., \& Pillar, G. (2012). Breastfeeding may improve nocturnal sleep and reduce infantile colic: Potential role of breast milk melatonin. European Journal of Pediatrics, 171, 729-732. doi:10.1007/s00431-0111659-3

Field, T. (2017). Infant sleep problems and interventions: A review. Infant Behavior and Development, 47, 40-53. doi:10. 1016/j.infbeh.2017.02.002

Figueiredo, B., Dias, C. C., Pinto, T. M., \& Field, T. (2016). Infant sleep-wake behaviors at two weeks, three and six months. Infant Behavior and Development, 44, 169-178. doi:10.1016/j.infbeh.2016.06.011

Fouts, H. N., Roopnarine, J. L., \& Lamb, M. E. (2007). Social experiences and daily routines of African American infants in different socioeconomic contexts. Journal of Family Psychology, 21, 655-664. doi:10.1037/0893-3200.21.4.655

Galbally, M., Lewis, A. J., McEgan, K., Scalzo, K., \& Islam, F. M. (2013). Breastfeeding and infant sleep patterns: An Australian population study. Journal of Paediatrics and Child Health, 49, E147-E152. doi:10.1111/jpc.12089

Gertner, S., Greenbaum, C. W., Sadeh, A., Dolfin, Z., Sirota, L., \& Ben-Nun, Y. (2002). Sleep-wake patterns in preterm infants and 6 month's home environment: Implications for early cognitive development. Early Human Development, 68, 93102. doi:10.1016/S0378-3782(02)00018-X

Ghaem, M., Armstrong, K. L., Trocki, O., Cleghorn, G. J., Patrick, M. K., \& Shepherd, R. W. (1998). The sleep patterns of infants and young children with gastro-oesophageal reflux. Journal of Paediatrics and Child Health, 34, 160-163. doi:10.1046/j. 1440-1754.1998.00191.x

Gibson, R., Elder, D., \& Gander, P. (2012). Actigraphic sleep and developmental progress of one-year-old infants. Sleep and Biological Rhythms, 10, 77-83. doi:10.1111/j.1479-8425.2011.00525.x

Gibson, R., Gander, P., \& Elder, D. (2012). Factors differentiating infants identified by parents as problem sleepers, and those that are not. Sleep and Biological Rhythms, 10, 46-52. doi:10.1111/j.1479-8425.2011.00517.x

Gillman, M. W., Rifas-Shiman, S. L., Kleinman, K., Oken, E., Rich-Edwards, J. W., \& Taveras, E. M. (2008). Developmental origins of childhood overweight: Potential public health impact. Obesity, 16, 1651-1656. doi:10.1038/oby.2008.260

Goldberg, W. A., Lucas-Thompson, R. G., Germo, G. R., Keller, M. A., Davis, E. P., \& Sandman, C. A. (2013). Eye of the beholder? Maternal mental health and the quality of infant sleep. Social Science \& Medicine, 79, 101-108. doi:10. 1016/j.socscimed.2012.07.006

Goodlin-Jones, B. L., Burnham, M. M., Gaylor, E. E., \& Anders, T. F. (2001). Night waking, sleep-wake organization, and selfsoothing in the first year of life. Journal of Developmental and Behavioral Pediatrics: JDBP, 22(4), 226-233.

Green, K. E., Groves, M. M., \& Tegano, D. W. (2004). Parenting practices that limit transitional object use: An illustration. Early Child Development and Care, 174, 427-436. doi:10.1080/0300443032000153606

Gress-Smith, J. L., Luecken, L. J., Lemery-Chalfant, K., \& Howe, R. (2012). Postpartum depression prevalence and impact on infant health, weight, and sleep in low-income and ethnic minority women and infants. Maternal and Child Health Journal, 16, 887-893. doi:10.1007/s10995-011-0812-y

Guyer, C., Huber, R., Fontijn, J., Bucher, H. U., Nicolai, H., Werner, H., ... Jenni, O. G. (2015). Very preterm infants show earlier emergence of 24-hour sleep-wake rhythms compared to term infants. Early Human Development, 91, 37-42. doi:10. 1016/j.earlhumdev.2014.11.002

Hanft, A., Burnham, M., Goodlin-Jones, B., \& Anders, T. F. (2006). Sleep Architecture in infants of Substance-Abusing mothers. Infant Mental Health Journal, 27, 141-151. doi:10.1002/imhj.20085

Harrison, Y. (2004). The relationship between daytime exposure to light and night-time sleep in 6-12-week-old infants. Journal of Sleep Research, 13, 345-352. doi:10.1111/j.1365-2869.2004.00435.x

Henderson, J. M., France, K. G., \& Blampied, N. M. (2011). The consolidation of infants' nocturnal sleep across the first year of life. Sleep Medicine Reviews, 15, 211-220. doi:10.1016/j.smrv.2010.08.003

Hiscock, H., Scalzo, K., Canterford, L., \& Wake, M. (2011). Sleep duration and body mass index in 0-7-year olds. Archives of Disease in Childhood, 96, 735-739. doi:10.1136/adc.2010.204925 
Hiscock, H., \& Wake, M. (2001). Infant sleep problems and postnatal depression: A community-based study. Pediatrics, 107, 1317-1322. doi:10.1542/peds.107.6.1317

Huang, Y. S., Paiva, T., Hsu, J. F., Kuo, M. C., \& Guilleminault, C. (2014). Sleep and breathing in premature infants at 6 months post-natal age. BMC Pediatrics, 14, 303. doi:10.1186/s12887-014-0303-6

Huang, X. N., Wang, H. S., Chang, J. J., Wang, L. H., Liu, X. C., Jiang, J. X., \& An, L. (2016). Feeding methods, sleep arrangement, and infant sleep patterns: A Chinese population-based study. World Journal of Pediatrics, 12, 66-75. doi:10.1007/ s12519-015-0012-8

Hughes, A., Gallagher, S., \& Hannigan, A. (2015). A cluster analysis of reported sleeping patterns of 9-month old infants and the association with maternal health: Results from a population based cohort study. Maternal and Child Health Journal, 19, 1881-1889. doi:10.1007/s10995-015-1701-6

Humphreys, J. S., Gringras, P., Blair, P. S., Scott, N., Henderson, J., Fleming, P. J., \& Emond, A. M. (2014). Sleep patterns in children with autistic spectrum disorders: A prospective cohort study. Archives of Disease in Childhood, 99, 114-118. doi:10.1136/archdischild-2013-304083

Hysing, M., Harvey, A. G., Torgersen, L., Ystrom, E., Reichborn-Kjennerud, T., \& Sivertsen, B. (2014). Trajectories and predictors of nocturnal awakenings and sleep duration in infants. Journal of Developmental \& Behavioral Pediatrics, 35, 309-316. doi:10.1097/DBP.0000000000000064

Jansen, P. W., Saridjan, N. S., Hofman, A., Jaddoe, V. W., Verhulst, F. C., \& Tiemeier, H. (2011). Does disturbed sleeping precede symptoms of anxiety or depression in toddlers? The generation R study. Psychosomatic Medicine, 73, 242249. doi:10.1097/PSY.0b013e31820a4abb

Jenni, O. G., Fuhrer, H. Z., Iglowstein, I., Molinari, L., \& Largo, R. H. (2005). A longitudinal study of bed sharing and sleep problems among Swiss children in the first 10 years of life. Pediatrics, 115, 233-240. doi:10.1542/peds.2004-0815E

Johnson, N., McMahon, C., \& Gibson, F. (2014). Assisted conception, maternal personality and parenting: Associations with toddler sleep behaviour. Journal of Paediatrics and Child Health, 50, 732-738. doi:10.1111/jpc.12654

Kaley, F., Reid, V., \& Flynn, E. (2012). Investigating the biographic, social and temperamental correlates of young infants' sleeping, crying and feeding routines. Infant Behavior and Development, 35, 596-605. doi:10.1016/j.infbeh.2012.03.004

Karraker, K. H., \& Young, M. (2007). Night waking in 6-month-old infants and maternal depressive symptoms. Journal of Applied Developmental Psychology, 28, 493-498. doi:10.1016/j.appdev.2007.06.002

Kirjavainen, J., Kirjavainen, T., Huhtala, V., Lehtonen, L., Korvenranta, H., \& Kero, P. (2001). Infants with colic have a normal sleep structure at 2 and 7 months of age. The Journal of Pediatrics, 138, 218-223. doi:10.1067/mpd.2001.110326

Klingenberg, L., Christensen, L. B., Hjorth, M. F., Zangenberg, S., Chaput, J. P., Sjödin, A., ... Michaelsen, K. F. (2013). No relation between sleep duration and adiposity indicators in 9-36 months old children: The SKOT cohort. Pediatric Obesity, 8, e14-e18. doi:10.1111/j.2047-6310.2012.00109.x

Konrad, C., Herbert, J. S., Schneider, S., \& Seehagen, S. (2016). The relationship between prior night's sleep and measures of infant imitation. Developmental Psychobiology, 58, 450-461. doi:10.1002/dev.21387

Korte, J., Hoehn, T., \& Siegmund, R. (2004). Actigraphic recordings of activity-rest rhythms of neonates born by different delivery modes. Chronobiology International, 21, 95-106. doi:10.1081/CBI-120027980

Kozyrskyj, A. L., Kendall, G. E., Zubrick, S. R., Newnham, J. P., \& Sly, P. D. (2009). Frequent nocturnal awakening in early life is associated with nonatopic asthma in children. European Respiratory Journal, 34, 1288-1295. doi:10.1183/09031936. 00040509

Küpers, L. K., L'Abée, C., Bocca, G., Stolk, R. P., Sauer, P. J., \& Corpeleijn, E. (2015). Determinants of weight gain during the first two years of life-The GECKO Drenthe birth cohort. PloS One, 10, e0133326. doi:10.1371/journal.pone.0133326

Lampl, M., \& Johnson, M. L. (2011). Infant growth in length follows prolonged sleep and increased naps. Sleep, 34, 641650. doi:10.1093/sleep/34.5.641

Landau, R., Sadeh, A., Vassoly, P., Berger, A., Atzaba-Poria, N., \& Auerbach, J. G. (2010). Sleep patterns of 7-week-old infants at familial risk for attention deficit hyperactivity disorder. Infant Mental Health Journal, 31, 630-646. doi:10.1002/imhj. 20275

Larson, M. C., White, B. P., Cochran, A., Donzella, B., \& Gunnar, M. (1998). Dampening of the cortisol response to handling at 3 months in human infants and its relation to sleep, circadian cortisol activity, and behavioral distress. Developmental Psychobiology, 33, 327-337. doi:10.1002/(SICI)1098-2302(199812)33:4>327::AID-DEV4>3.0.CO;2-S

Lee, K. (2000). Crying and behavior pattern in breast- and formula-fed infants. Early Human Development, 58, $133-140$. doi:10.1016/S0378-3782(00)00071-2

Lehnkering, H., Korte, J., \& Siegmund, R. (2009). Activity-rest rhythm of term-born neonates exposed to nicotine during pregnancy. Biological Rhythm Research, 40, 145-151. doi:10.1080/09291010802379204

Loutzenhiser, L., McAuslan, P., \& Sharpe, D. P. (2015). The trajectory of maternal and paternal fatigue and factors associated with fatigue across the transition to parenthood. Clinical Psychologist, 19, 15-27. doi:10.1111/cp.12048

Lucas-Thompson, R., Goldberg, W. A., Germo, G. R., Keller, M. A., Davis, E. P., \& Sandman, C. A. (2009). Sleep arrangements and night waking at 6 and 12 months in relation to infants' stress-induced cortisol responses. Infant and Child Development, 18, 521-544. doi:10.1002/icd.636

Lukowski, A. F., Liu, X., Peirano, P., Odio, M., \& Bauer, P. J. (2015). Disposable diaper use promotes consolidated nighttime sleep and positive mother-infant interactions in Chinese 6-month-olds. Journal of Family Psychology, 29, 371-381. doi:10.1037/fam0000072 
Lukowski, A. F., \& Milojevich, H. M. (2013). Sleeping like a baby: Examining relations between habitual infant sleep, recall memory, and generalization across cues at 10 months. Infant Behavior and Development, 36, 369-376. doi:10.1016/j. infbeh.2013.02.001

Mao, A., Burnham, M. M., Goodlin-Jones, B. L., Gaylor, E. E., \& Anders, T. F. (2004). A comparison of the sleep-wake patterns of cosleeping and solitary-sleeping infants. Child Psychiatry and Human Development, 35, 95-105. doi:10.1007/s10578004-1879-0

Mao, Q., Zhu, L. X., \& Su, X. Y. (2011). A comparison of postnatal depression and related factors between Chinese new mothers and fathers. Journal of Clinical Nursing, 20, 645-652. doi:10.1111/j.1365-2702.2010.03542.x

McDaniel, B. T., \& Teti, D. M. (2012). Coparenting quality during the first three months after birth: The role of infant sleep quality. Journal of Family Psychology, 26, 886-895. doi:10.1037/a0030707

McGeorge, K., Milne, L., Cotton, L., \& Whelan, T. (2015). Effects of infant and maternal sensory processing on infant fussing, crying, and sleep. Infant Mental Health Journal, 36, 275-286. doi:10.1002/imhj.21510

McKay, S. M., \& Angulo-Barroso, R. M. (2006). Longitudinal assessment of leg motor activity and sleep patterns in infants with and without Down syndrome. Infant Behavior and Development, 29, 153-168. doi:10.1016/j.infbeh.2005.09.004

Mindell, J. A., Du Mond, C., Tanenbaum, J. B., \& Gunn, E. (2012). Long-term relationship between breastfeeding and sleep. Children's Health Care, 41, 190-203. doi:10.1080/02739615.2012.685038

Mindell, J. A., \& Lee, C. (2015). Sleep, mood, and development in infants. Infant Behavior and Development, 41, $102-107$. doi:10.1016/j.infbeh.2015.08.004

Mindell, J. A., Meltzer, L. J., Carskadon, M. A., \& Chervin, R. D. (2009). Developmental aspects of sleep hygiene: Findings from the 2004 national sleep foundation sleep in America Poll. Sleep Medicine, 10, 771-779. doi:10.1016/j.sleep.2008. 07.016

Mindell, J. A., Sadeh, A., Wiegand, B., How, T. H., \& Goh, D. Y. (2010). Cross-cultural differences in infant and toddler sleep. Sleep Medicine, 11, 274-280. doi:10.1016/j.sleep.2009.04.012

Moher, D., Liberati, A., Tetzlaff, J., Altman, D. G., \& Prisma Group. (2009). Preferred reporting items for systematic reviews and meta-analyses: The PRISMA statement. PLoS Medicine, 6, e1000097. doi:10.1371/journal.pmed.1000097

Möllborg, P., Wennergren, G., Norvenius, S. G., \& Alm, B. (2011). Bed-sharing among six-month-old infants in western Sweden. Acta Paediatrica, 100, 226-230. doi:10.1111/j.1651-2227.2010.02008.x

Morgan, J. B., Lucas, A., \& Fewtrell, M. S. (2004). Does weaning influence growth and health up to 18 months? Archives of Disease in Childhood, 89, 728-733. doi:10.1136/adc.2003.036137

Morrell, J., \& Steele, H. (2003). The role of attachment security, temperament, maternal perception, and care-giving behavior in persistent infant sleeping problems. Infant Mental Health Journal, 24, 447-468. doi:10.1002/imhj.10072

Nevarez, M. D., Rifas-Shiman, S. L., Kleinman, K. P., Gillman, M. W., \& Taveras, E. M. (2010). Associations of early life risk factors with infant sleep duration. Academic Pediatrics, 10, 187-193. doi:10.1016/j.acap.2010.01.007

Novosad, C., Freudigman, K., \& Thoman, E. B. (1999). Sleep patterns in newborns and temperament at eight months: A preliminary study. Journal of Developmental \& Behavioral Pediatrics, 20, 99-105. doi:10.1097/00004703-19990400000005

O'Connor, T. G., Caprariello, P., Blackmore, E. R., Gregory, A. M., Glover, V., Fleming, P., \& ALSPAC Study Team. (2007). Prenatal mood disturbance predicts sleep problems in infancy and toddlerhood. Early Human Development, 83, 451-458. doi:10.1016/j.earlhumdev.2006.08.006

Odoi, A., Andrew, S., Wong, F. Y., Yiallourou, S. R., \& Horne, R. S. (2014). Pacifier use does not alter sleep and spontaneous arousal patterns in healthy term-born infants. Acta Paediatrica, 103, 1244-1250. doi:10.1111/apa.12790

Orsi, K. C. S. C., Llaguno, N. S., Avelar, A. F. M., Tsunemi, M. H., Pedreira, M. D. L. G., Sato, M. H., \& Pinheiro, E. M. (2015). Effect of reducing sensory and environmental stimuli during hospitalized premature infant sleep. Revista da Escola de Enfermagem da USP, 49, 0550-0555. doi:10.1590/S0080-623420150000400003

Palmstierna, P., Sepa, A., \& Ludvigsson, J. (2008). Parent perceptions of child sleep: A study of 10000 Swedish children. Acta Paediatrica, 97, 1631-1639. doi:10.1111/j.1651-2227.2008.00967.x

Pellegrini-Belinchón, J., Miguel-Miguel, G., De Dios-Martin, B., Vicente-Galindo, E., Lorente-Toledano, F., \& Garcia-Marcos, L. (2012). Study of wheezing and its risk factors in the first year of life in the Province of Salamanca, Spain. The EISL study. Allergologia et Immunopathologia, 40, 164-171. doi:10.1016/j.aller.2011.03.014

Pennestri, M. H., Moss, E., O'Donnell, K., Lecompte, V., Bouvette-Turcot, A. A., Atkinson, L., ... Gaudreau, H. (2015). Establishment and consolidation of the sleep-wake cycle as a function of attachment pattern. Attachment \& Human Development, 17, 23-42. doi:10.1080/14616734.2014.953963

Philbrook, L. E., \& Teti, D. M. (2016). Bidirectional associations between bedtime parenting and infant sleep: Parenting quality, parenting practices, and their interaction. Journal of Family Psychology, 30(431), doi:10.1037/fam0000198

Piteo, A. M., Roberts, R. M., Nettelbeck, T., Burns, N., Lushington, K., Martin, A. J., \& Kennedy, J. D. (2013). Postnatal depression mediates the relationship between infant and maternal sleep disruption and family dysfunction. Early Human Development, 89, 69-74. doi:10.1016/j.earlhumdev.2012.07.017

Quillin, S. I. (1997). Infant and mother sleep patterns during 4th postpartum week. Issues in Comprehensive Pediatric Nursing, 20, 115-123. doi:10.3109/01460869709026882

Quillin, S. I., \& Glenn, L. L. (2004). Interaction between feeding method and co-sleeping on maternal-newborn sleep. Journal of Obstetric, Gynecologic, \& Neonatal Nursing, 33, 580-588. doi:10.1177/0884217504269013 
Ramamurthy, M. B., Sekartini, R., Ruangdaraganon, N., Huynh, D. H. T., Sadeh, A., \& Mindell, J. A. (2012). Effect of current breastfeeding on sleep patterns in infants from Asia-Pacific region. Journal of Paediatrics and Child Health, 48, 669-674. doi:10.1111/j.1440-1754.2012.02453.x

Sadeh, A., De Marcas, G., Guri, Y., Berger, A., Tikotzky, L., \& Bar-Haim, Y. (2015). Infant sleep predicts attention regulation and behavior problems at 3-4 years of age. Developmental Neuropsychology, 40, 122-137. doi:10.1080/87565641.2014. 973498

Sadeh, A., Tikotzky, L., \& Scher, A. (2010). Parenting and infant sleep. Sleep Medicine Reviews, 14, 89-96. doi:10.1016/j.smrv. 2009.05.003

Saenz, J., Yaugher, A., \& Alexander, G. M. (2015). Sleep in infancy predicts gender specific social-emotional problems in toddlers. Frontiers in Pediatrics, 3, 1-6. doi:10.3389/fped.2015.00042

Santos, I. S., Matijasevich, A., \& Domingues, M. R. (2012). Maternal caffeine consumption and infant nighttime waking: Prospective cohort study. Pediatrics, 129, 860-868. doi:10.1542/peds.2011-1773

Santos, I. S., Mota, D. M., \& Matijasevich, A. (2008). Epidemiology of co-sleeping and nighttime waking at 12 months in a birth cohort. Jornal de Pediatria, 84, 114-122. doi:10.1590/S0021-75572008000200005

Scher, A. (2001a). Attachment and sleep: A study of night waking in 12-month-old infants. Developmental Psychobiology, 38, 274-285. doi:10.1002/dev.1020

Scher, A. (2001b). Mother-child interaction and sleep regulation in one-year-olds. Infant Mental Health Journal, 22, 515528. doi:10.1002/imhj.1015

Scher, A. (2005a). Crawling in and out of sleep. Infant and Child Development, 14, 491-500. doi:10.1002/icd.427

Scher, A. (2005b). Infant sleep at 10 months of age as a window to cognitive development. Early Human Development, 81 , 289-292. doi:10.1016/j.earlhumdev.2004.07.005

Scher, A. (2008). Maternal separation anxiety as a regulator of infants' sleep. Journal of Child Psychology and Psychiatry, 49, 618-625. doi:10.1111/j.1469-7610.2007.01872.x

Scher, A., \& Blumberg, O. (1999). Night waking among 1-year olds: A study of maternal separation anxiety. Child: Care, Health and Development, 25, 323-334. doi:10.1046/j.1365-2214.1999.00099.x

Scher, A., \& Cohen, D. (2005). Locomotion and nightwaking. Child: Care, Health and Development, 31, 685-691. doi:10. 1111/j.1365-2214.2005.00557.x

Scher, A., \& Cohen, D. (2015). V. Sleep as a Mirror of developmental Transitions in infancy: The case of crawling. Monographs of the Society for Research in Child Development, 80, 70-88. doi:10.1111/mono.12145

Scher, A., Tirosh, E., \& Lavie, P. (1998). The relationship between sleep and temperament revisited: Evidence for 12-montholds: A research note. The Journal of Child Psychology and Psychiatry and Allied Disciplines, 39(5), 785-788.

Schwichtenberg, A. J., Anders, T. F., Vollbrecht, M., \& Poehlmann, J. (2011). Daytime sleep and parenting interactions in infants born preterm. Journal of Developmental and Behavioral Pediatrics: JDBP, 32, 8-17. doi:10.1097/DBP. 0b013e3181fa57e4

Schwichtenberg, A. J. M., \& Poehlmann, J. (2009). A transactional model of sleep-wake regulation in infants born preterm or low birthweight. Journal of Pediatric Psychology, 34, 837-849. doi:10.1093/jpepsy/jsn132

Schwichtenberg, A. J., Shah, P. E., \& Poehlmann, J. (2013). Sleep and attachment in preterm infants. Infant Mental Health Journal, 34, 37-46. doi:10.1002/imhj.21374

Scott, N., Blair, P. S., Emond, A. M., Fleming, P. J., Humphreys, J. S., Henderson, J., \& Gringras, P. (2013). Sleep patterns in children with ADHD: A population-based cohort study from birth to 11 years. Journal of Sleep Research, 22, $121-128$. doi:10.1111/j.1365-2869.2012.01054.x

Sharkey, K. M., Iko, I. N., Machan, J. T., Thompson-Westra, J., \& Pearlstein, T. B. (2016). Infant sleep and feeding patterns are associated with maternal sleep, stress, and depressed mood in women with a history of major depressive disorder (MDD). Archives of Women's Mental Health, 19, 209-218. doi:10.1007/s00737-015-0557-5

Shibagaki, M., Sawata, T., \& Tachibana, T. (2004). Relation between polysomnographic measures during nocturnal sleep and a quotient of behavioral development in infants with developmental disabilities. Perceptual and Motor Skills, 99, 429-434. doi:10.2466/pms.99.2.429-434

Shinohara, H., \& Kodama, H. (2011). Relationship between circadian salivary melatonin levels and sleep-wake behavior in infants. Pediatrics International, 53, 29-35. doi:10.1111/j.1442-200X.2010.03186.x

Sinai, D., \& Tikotzky, L. (2012). Infant sleep, parental sleep and parenting stress in families of mothers on maternity leave and in families of working mothers. Infant Behavior and Development, 35, 179-186. doi:10.1016/j.infbeh.2012.01.006

So, K., Michael Adamson, T., \& Horne, R. S. (2007). The use of actigraphy for assessment of the development of sleep/wake patterns in infants during the first 12 months of life. Journal of Sleep Research, 16, 181-187. doi:10.1111/j.1365-2869. 2007.00582.x

Sorondo, B. M., \& Reeb-Sutherland, B. C. (2015). Associations between infant temperament, maternal stress, and infants sleep across the first year of life. Infant Behavior and Development, 39, 131-135. doi:10.1016/j.infbeh.2015.02.010

Spruyt, K., Aitken, R. J., So, K., Charlton, M., Adamson, T. M., \& Horne, R. S. (2008). Relationship between sleep/wake patterns, temperament and overall development in term infants over the first year of life. Early Human Development, 84, 289-296. doi:10.1016/j.earlhumdev.2007.07.002

St James-Roberts, I., Conroy, S., \& Hurry, J. (1997). Links between infant crying and sleep-waking at six weeks of age. Early Human Development, 48, 143-152. doi:10.1016/S0378-3782(96)01845-2 
St James-Roberts, I., \& Peachey, E. (2011). Distinguishing infant prolonged crying from sleep-waking problems. Archives of Disease in Childhood, 96, 340-344. doi:10.1136/adc.2010.200204

Telliez, F., Bach, V., Leke, A., Chardon, K., \& Libert, J. P. (2002). Feeding behavior in neonates whose diet contained medium-chain triacylglycerols: Short-term effects on thermoregulation and sleep. The American Journal of Clinical Nutrition, 76(5), 1091-1095.

Thomas, K. A. (2000). Differential effects of breast- and formula-Feeding on preterm infants' sleep-wake patterns. Journal of Obstetric, Gynecologic, \& Neonatal Nursing, 29, 145-152. doi:10.1111/j.1552-6909.2000.tb02034.x

Thomas, K. A., \& Foreman, S. W. (2005). Infant sleep and feeding pattern: Effects on maternal sleep. Journal of Midwifery \& Women's Health, 50, 399-404. doi:10.1016/j.jmwh.2005.04.010

Thomas, K. A., \& Spieker, S. (2016). Sleep, depression, and fatigue in late postpartum. MCN. The American Journal of Maternal Child Nursing, 41, 104-109. doi:10.1097/NMC.0000000000000213

Tikotzky, L., De Marcas, G., Har-Toov, J., Dollberg, S., Bar-Haim, Y., \& Sadeh, A. (2010). Sleep and physical growth in infants during the first 6 months. Journal of Sleep Research, 19, 103-110. doi:10.1111/j.1365-2869.2009.00772.x

Tikotzky, L., \& Sadeh, A. (2009). Maternal sleep-Related cognitions and infant sleep: A Longitudinal study from pregnancy through the 1st year. Child Development, 80, 860-874. doi:10.1111/j.1467-8624.2009.01302.x

Tikotzky, L., \& Sadeh, A. (2010). The role of cognitive-behavioral therapy in behavioral childhood insomnia. Sleep Medicine, 11, 686-691. doi:10.1016/j.sleep.2009.11.017

Tikotzky, L., Sadeh, A., \& Glickman-Gavrieli, T. (2010). Infant sleep and paternal involvement in infant caregiving during the first 6 months of life. Journal of Pediatric Psychology, 36, 36-46. doi:10.1093/jpepsy/jsq036

Tikotzky, L., Sadeh, A., Volkovich, E., Manber, R., Meiri, G., \& Shahar, G. (2015). VII. Infant sleep development from 3 to 6 months postpartum: Links with maternal sleep and paternal involvement. Monographs of the Society for Research in Child Development, 80, 107-124. doi:10.1111/mono.12147

Tikotzky, L., \& Shaashua, L. (2012). Infant sleep and early parental sleep-related cognitions predict sleep in pre-school children. Sleep Medicine, 13, 185-192. doi:10.1016/j.sleep.2011.07.013

Tomalski, P., Moore, D. G., Ribeiro, H., Axelsson, E. L., Murphy, E., Karmiloff-Smith, A., ... Kushnerenko, E. (2013). Socioeconomic status and functional brain development - Associations in early infancy. Developmental Science, 16, 676-687. doi:10.1111/desc.12079

Touchette, E., Dionne, G., Forget-Dubois, N., Petit, D., Pérusse, D., Falissard, B., ... Montplaisir, J. Y. (2013). Genetic and environmental influences on daytime and nighttime sleep duration in early childhood. Pediatrics, 131, e1874e1880. doi:10.1542/peds.2012-2284

Touchette, É, Petit, D., Paquet, J., Boivin, M., Japel, C., Tremblay, R. E., \& Montplaisir, J. Y. (2005). Factors associated with fragmented sleep at night across early childhood. Archives of Pediatrics \& Adolescent Medicine, 159, 242-249. doi:10. 1001/archpedi.159.3.242

Tourula, M., Isola, A., Hassi, J., Bloigu, R., \& Rintamäki, H. (2010). Infants sleeping outdoors in a northern winter climate: Skin temperature and duration of sleep. Acta Paediatrica, 99, 1411-1417. doi:10.1111/j.1651-2227.2010.01814.x

Utkun, E., Öndoğan, Z., Yalaz, M., \& Yıldırım Sözmen, E. (2015). The effect of different types of fabric on the clothing comfort, sleep pattern and saliva cortisol \& melatonin levels of infants. Journal of Textile Apparel, 25, 119-124.

Vijakkhana, N., Wilaisakditipakorn, T., Ruedeekhajorn, K., Pruksananonda, C., \& Chonchaiya, W. (2015). Evening media exposure reduces night-time sleep. Acta Paediatrica, 104, 306-312. doi:10.1111/apa.12904

Volkovich, E., Ben-Zion, H., Karny, D., Meiri, G., \& Tikotzky, L. (2015). Sleep patterns of co-sleeping and solitary sleeping infants and mothers: A longitudinal study. Sleep Medicine, 16, 1305-1312. doi:10.1016/j.sleep.2015.08.016

Warren, S. L., Howe, G., Simmens, S. J., \& Dahl, R. E. (2006). Maternal depressive symptoms and child sleep: Models of mutual influence over time. Development and Psychopathology, 18(1), 1-16. doi:10.1017/S0954579406060019

Weinraub, M., Bender, R. H., Friedman, S. L., Susman, E. J., Knoke, B., Bradley, R., .. Williams, J. (2012). Patterns of developmental change in infants' nighttime sleep awakenings from 6 through 36 months of age. Developmental Psychology, 48, 1511-1528. doi:10.1037/a0027680

White, B. P., Gunnar, M. R., Larson, M. C., Donzella, B., \& Barr, R. G. (2000). Behavioral and physiological responsivity, sleep, and patterns of daily cortisol production in infants with and without colic. Child Development, 71, 862-877. doi:10. 1111/1467-8624.00196

Wolke, D., Söhne, B., Riegel, K., Ohrt, B., \& Österlund, K. (1998). An epidemiologic longitudinal study of sleeping problems and feeding experience of preterm and term children in southern Finland: Comparison with a southern German population sample. The Journal of Pediatrics, 133, 224-231. doi:10.1016/S0022-3476(98)70224-0

Zaidman-Zait, A., \& Hall, W. A. (2015). Children's night waking among toddlers: Relationships with mothers' and fathers' parenting approaches and children's behavioural difficulties. Journal of Advanced Nursing, 71, 1639-1649. doi:10.1111/ jan.12636

Zentall, S. R., Braungart-Rieker, J. M., Ekas, N. V., \& Lickenbrock, D. M. (2012). Longitudinal assessment of sleep-wake regulation and attachment security with parents. Infant and Child Development, 21, 443-457. doi:10.1002/icd.175 